\title{
HIGH-PRECISION ORBITAL AND PHYSICAL PARAMETERS OF DOUBLE-LINED SPECTROSCOPIC BINARY STARS—HD78418, HD123999, HD160922, HD200077, AND HD210027
}

\author{
Maciej Konacki ${ }^{1,2}$, Matthew W. Muterspaugh ${ }^{3,4}$, Shrinivas R. Kulkarni ${ }^{5}$, and Krzysztof G. Hęminiak ${ }^{1}$ \\ ${ }^{1}$ Nicolaus Copernicus Astronomical Center, Polish Academy of Sciences, Rabianska 8, 87-100 Torun, Poland; maciej@ncac.torun.pl \\ ${ }^{2}$ Astronomical Observatory, A. Mickiewicz University, Sloneczna 36, 60-286 Poznan, Poland \\ ${ }^{3}$ Department of Mathematics and Physics, College of Arts and Sciences, Tennessee State University, Boswell Science Hall, Nashville, TN 37209 , USA \\ ${ }^{4}$ Tennessee State University, Center of Excellence in Information Syste3500 John A. Merritt Blvd., Box No. 9501, Nashville, TN 37203-3401, USA \\ ${ }^{5}$ Division of Physics, Mathematics and Astronomy, California Institute of Technology, Pasadena, CA 91125, USA \\ Received 2009 October 23; accepted 2010 June 16; published 2010 July 28
}

\begin{abstract}
We present high-precision radial velocities (RVs) of double-lined spectroscopic binary stars HD78418, HD123999, HD160922, HD200077, and HD210027. They were obtained based on the high-resolution echelle spectra collected with the Keck I/HIRES, Shane/CAT/Hamspec, and TNG/Sarge telescopes/spectrographs over the years 2003-2008 as part of the TATOOINE search for circumbinary planets. The RVs were computed using our novel iodine cell technique for double-line binary stars, which relies on tomographically disentangled spectra of the components of the binaries. The precision of the RVs is of the order of $1-10 \mathrm{~m} \mathrm{~s}^{-1}$, and to properly model such measurements one needs to account for the light-time effect within the binary's orbit, relativistic effects, and RV variations due to tidal distortions of the components of the binaries. With such proper modeling, our RVs combined with the archival visibility measurements from the Palomar Testbed Interferometer (PTI) allow us to derive very precise spectroscopic/astrometric orbital and physical parameters of the binaries. In particular, we derive the masses, the absolute $K$ - and $H$-band magnitudes, and the parallaxes. The masses together with the absolute magnitudes in the $K$ and $H$ bands enable us to estimate the ages of the binaries. These RVs allow us to obtain some of the most accurate mass determinations of binary stars. The fractional accuracy in $m$ sin $i$ only, and hence based on the RVs alone, ranges from $0.02 \%$ to $0.42 \%$. When combined with the PTI astrometry, the fractional accuracy in the masses in the three best cases ranges from $0.06 \%$ to $0.5 \%$. Among them, the masses of HD210027 components rival in precision the mass determination of the components of the relativistic double pulsar system PSR J0737-3039. In the near future, for double-lined eclipsing binary stars we expect to derive masses with a fractional accuracy of the order of up to $\sim 0.001 \%$ with our technique. This level of precision is an order of magnitude higher than of the most accurate mass determination for a body outside the solar system - the double neutron star system PSR B1913+16.
\end{abstract}

Key words: binaries: spectroscopic - stars: fundamental parameters - stars: individual (HD78418, HD123999, HD160922, HD200077, and HD210027) - techniques: radial velocities

Online-only material: color figures, machine-readable table

\section{INTRODUCTION}

The first observations of a spectroscopic binary star, $\zeta \mathrm{UMa}$ (Mizar), were announced by Edward C. Pickering (1846-1919) on 1889 November 13 during a meeting of the National Academy of Sciences in Philadelphia (Pickering 1890). A similar announcement about $\beta$ Per (Algol) was made by Herman C. Vogel (1841-1907) on 1889 November 28 during a session of Konglich-Preussiche Akademie der Wissenschaften (Vogel 1890a, 1890b). Even though a transatlantic telegraph cable had been available since the $1860 \mathrm{~s}$, it was quite unusual timing for a pre-astro-ph era. Pickering noted that the $\mathrm{K}$ line of Mizar occasionally appeared double and this way discovered the first double-lined spectroscopic binary star. Vogel measured radial velocities (RVs) of Algol and used them to prove that the known variations in the brightness of Algol are indeed caused by a "dark satellite revolving about it" (Vogel 1890b).

Vogel and collaborators built a series of prism spectrographs for the $30 \mathrm{~cm}$ refractor of the Potsdam Astrophysical Observatory (Vogel 1900). In 1888, they initiated a photographic RV program. Vogel was not the first to take a photograph of a stellar spectrum but improved the technique and obtained an RV precision of 2-4 $\mathrm{km} \mathrm{s}^{-1}$ (Vogel 1891). Vogel (in 1906) and Pickering (in 1908) were both awarded the Bruce medal. Yet another Bruce medalist (in 1915), William W. Campbell
(1862-1938) and collaborators carried out a large spectroscopic program at the Lick Observatory and discovered many spectroscopic binaries (Campbell et al. 1911). They prepared the first catalog of spectroscopic binaries (Campbell \& Curtis 1905). Vogel was the director of the Potsdam Observatory for 25 years, Pickering of the Harvard College Observatory for 42 years, and Campbell of the Lick Observatory for 30 years. The administrative duties must have been not too distracting those days.

Both Vogel and Campbell recognized the importance of flexure and temperature control of a spectrograph (Vogel 1890c; Campbell 1898, 1900), and Campbell also noted the issue of a slit illumination and its impact on RV precision (Campbell 1916). Today a high RV precision is achieved by either using a very stable fiber-fed spectrograph that is contained in a controlled environment (a vacuum tank) or using an absorption cell to superimpose a reference spectrum onto a stellar spectrum and this way measure and account for the systematic RV errors. Current state-of-the-art precision is at the level of $\sim 1 \mathrm{~m} \mathrm{~s}^{-1}$. It is however important to note that such a precision refers to single stars or at best single-lined spectroscopic binaries where the influence of the secondary spectrum can be neglected. In such a case, given a stable spectrograph, an RV measurement is essentially a measurement of a shift of an otherwise constant shape (spectrum). 


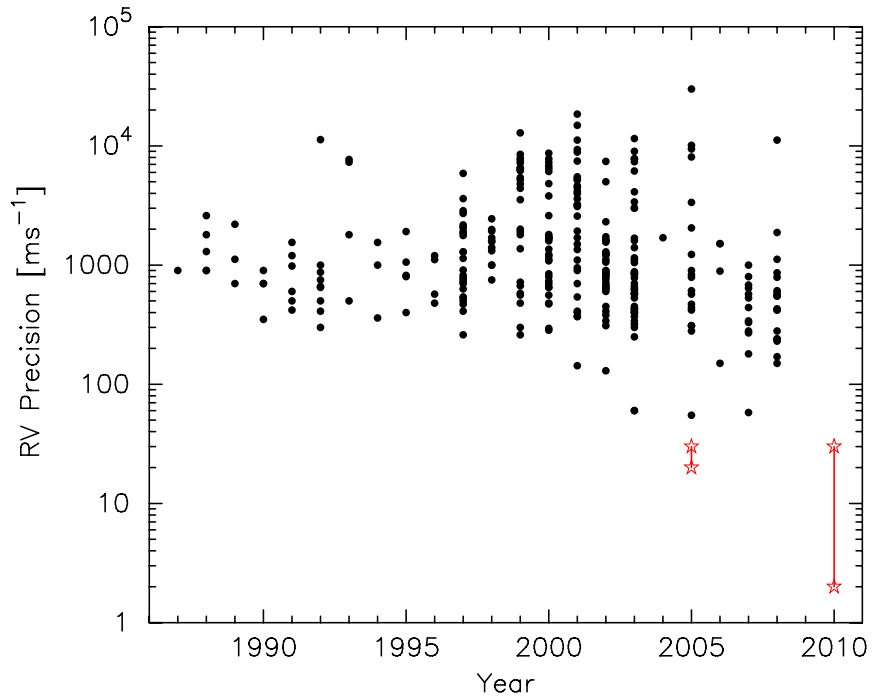

Figure 1. Radial velocity precision (an rms from the orbital fit) for the primaries of double-lined spectroscopic binaries as a function of the publication date based on the ninth catalog of spectroscopic binary orbits (Pourbaix et al. 2004). It is worth noting that already in the early 20th century an RV precision of several $\mathrm{km} \mathrm{s}^{-1}$ for double-lined binaries was possible (Plummer et al. 1908, HD83808). These RV measurements were typically obtained in the visible. There is however one case by Mazeh et al. (2003) where an RV precision of $\sim 100 \mathrm{~m} \mathrm{~s}^{-1}$ for SB2s was obtained in the infrared. Current and previous precision range from our method is delimited with the red stars (note the logarithmic scale).

(A color version of this figure is available in the online journal.)

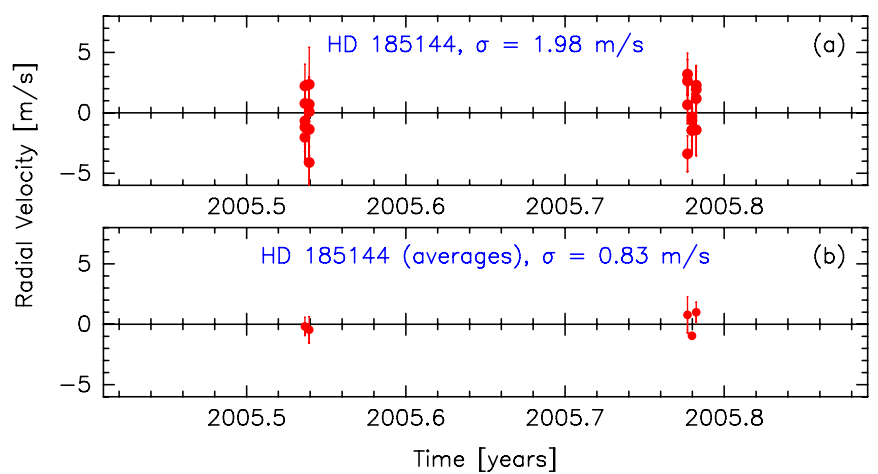

Figure 2. Series of the precision RV measurements of a single star HD185144 $(V=4.7 \mathrm{mag} ; \mathrm{K} 0 \mathrm{~V})$ taken with the Keck I/HIRES and reduced using our iodine cell data pipeline. The data were taken on five nights: two in July (five consecutive spectra each night) and three in 2005 October (four consecutive spectra each night). The average $\mathrm{S} / \mathrm{N}$ was 600 for the template exposure as well as for all the spectra taken with the iodine cell.

(A color version of this figure is available in the online journal.)

RVs of double-lined spectroscopic binary stars (SB2s) can be used effectively to derive basic parameters of stars if the stars happen to be eclipsing or their astrometric relative orbit can be determined. It is quite surprising that the RV precision of double-lined binary stars on average has not improved much over the last 100 years (see Figure 1). With the exception of our previous work (Konacki 2005, 2009), the RV precision for such targets typically varies from $\sim 0.1 \mathrm{~km} \mathrm{~s}^{-1}$ to $\sim 1 \mathrm{~km} \mathrm{~s}^{-1}$ and clearly is much worse than what has been achieved for stars with planets or single-lined binary stars. The main problem with double-lined binary stars is that one has to deal with two sets of superimposed spectral lines whose corresponding RVs change considerably with typical amplitudes of $\sim 50-100 \mathrm{~km} \mathrm{~s}^{-1}$. In consequence, a spectrum is highly variable and obviously one cannot measure RVs by noting a simple shift.

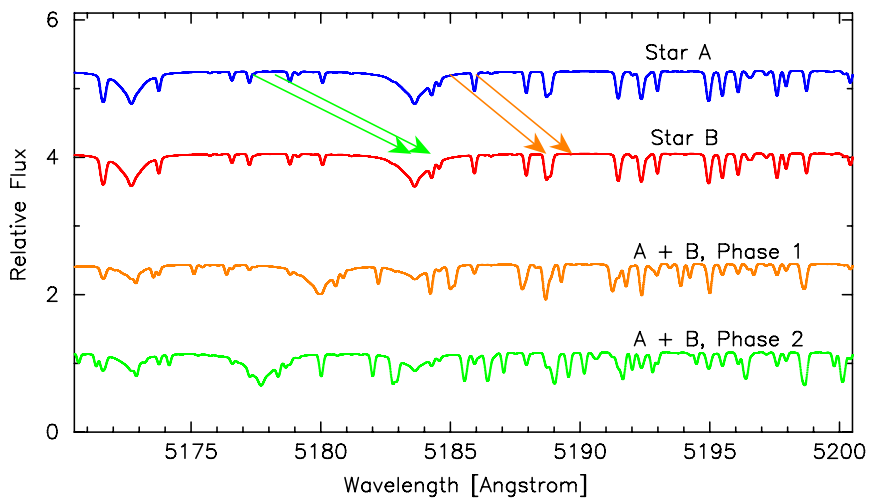

Figure 3. SB2 as nature's realization of the tomographic imaging. The composite spectrum of an SB2 taken at any orbital phase can be interpreted as a result of imaging of a two-layered object. By observing an SB2 at several different orbital phases and hence different RVs of its components, one can carry out an equivalent of tomographic imaging and eventually be able to disentangle the component ("layer") spectra.

(A color version of this figure is available in the online journal.)

We have developed a novel iodine cell based approach that employs a tomographic disentangling of the component spectra of SB2s and allows one to measure RVs of the components of SB2s with a precision of the order $1-10 \mathrm{~m} \mathrm{~s}^{-1}$ (Konacki 2009; Konacki et al. 2009). Such quality RVs not only enable us to search for circumbinary extrasolar planets (Konacki et al. 2009) but also to determine basic parameters of stars with an unprecedented precision. In particular, the masses of stars for noneclipsing SB2s can easily be determined with a fractional accuracy on the order of at least $\sim 0.1 \%$ and often even $\sim 0.01 \%$. Moreover, we expect that the accuracy in masses will reach the $\sim 0.001 \%$ level when our method is applied to eclipsing binary stars. Such a level of precision is an order of magnitude higher than of the most accurate mass determination for a body outside the solar system - the double neutron star system PSR B1913+16 (Nice et al. 2008).

Below we present our precision RV data sets for five targets HD78418, HD123999, HD160922, HD200077, and HD210027 from our ongoing TATOOINE (The Attempt To Observe Outerplanets In Non-single-stellar Environments) RV program to search for circumbinary planets. All of them have been extensively observed with the Palomar Testbed Interferometer (PTI; Colavita et al. 1999). The archival PTI visibility measurements can be used to derive relative astrometric orbits of the binaries. These combined with our spectroscopic orbits allow for a complete orbital and physical description of the systems (with the exception of the radii of the components). In Section 2, we describe the RV measurements and their modeling and in Section 3 the visibility measurements and their modeling. In Section 4, we present the spectroscopic and astrometric orbital solutions and the resulting orbital and physical parameters of the binaries. A discussion is provided in Section 5.

\section{RADIAL VELOCITIES}

\subsection{Iodine Absorption Cell and Spectroscopic Binary Stars}

In the iodine cell $\left(\mathrm{I}_{2}\right)$ technique, the Doppler shift of a star spectrum $\Delta \lambda$ is determined by solving the following equation (Marcy \& Butler 1992):

$$
I(\lambda)=\left[F\left(\lambda+\Delta \lambda_{s}\right) T\left(\lambda+\Delta \lambda_{\mathrm{I}_{2}}\right)\right] \otimes \mathrm{PSF},
$$



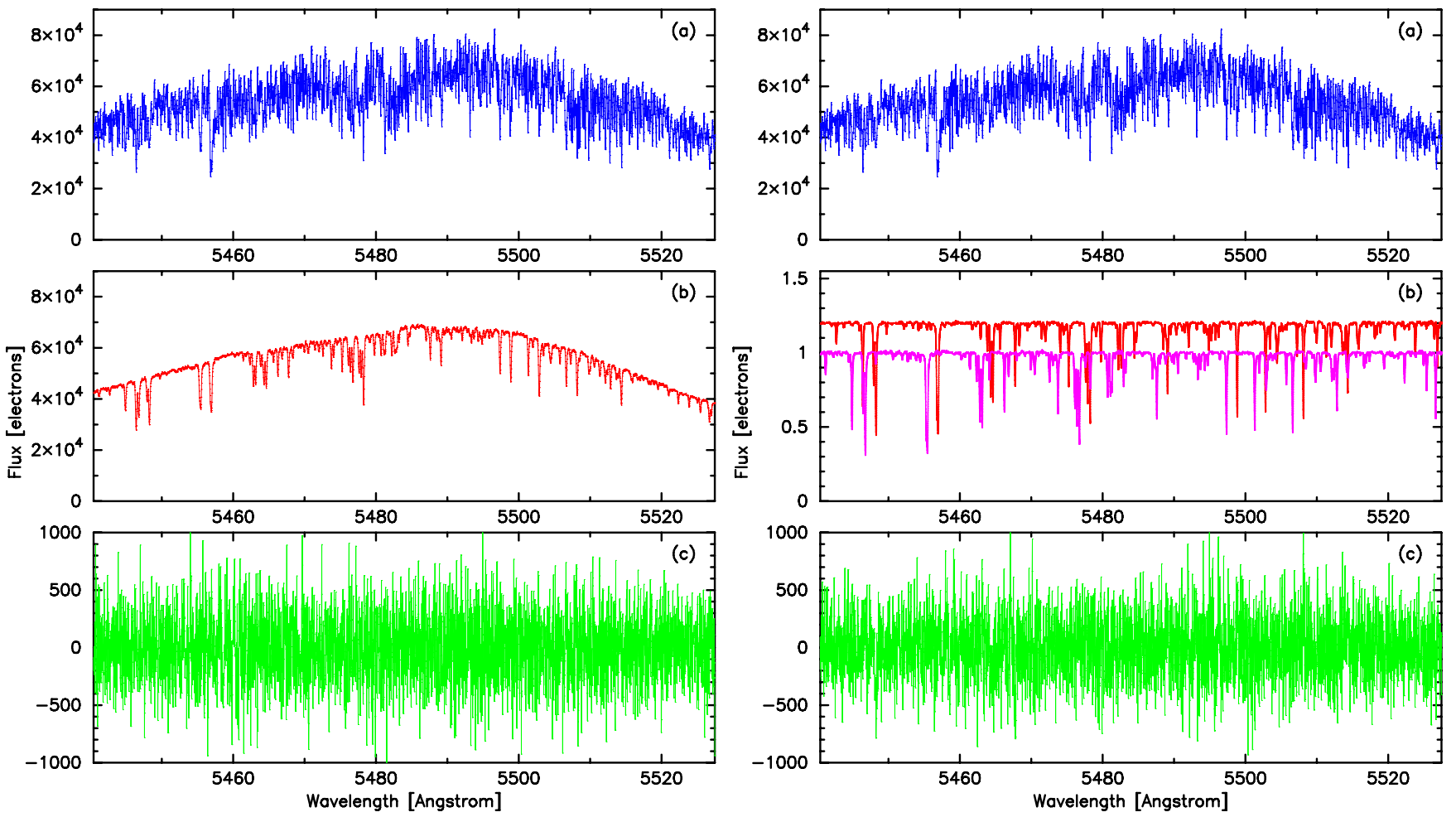

Figure 4. Left and right (a): a piece of a spectrum of HD4676 as a function of wavelength as seen through an iodine cell. Left (b): a template composite spectrum of HD4676 taken just after the iodine cell exposure shown in (a). Right (b): the corresponding continuum normalized disentangled component spectra of HD4676 shifted accordingly to their respective RVs. Left and right (c): the residuals after subtracting (b) from (a) and accounting for the iodine cell lines. The residuals from the disentangled component spectra are smaller than from the observed composite spectrum.

(A color version of this figure is available in the online journal.)

Table 1

Radial Velocities of HD78418, HD123999, HD160922, HD200077, and HD210027

\begin{tabular}{|c|c|c|c|c|c|c|c|c|c|c|}
\hline Target & $\begin{array}{c}\text { Time } \\
\text { (TDB-2400000.5) } \\
\end{array}$ & $\begin{array}{c}\mathrm{RV}_{1} \\
\left(\mathrm{~km} \mathrm{~s}^{-1}\right) \\
\end{array}$ & $\begin{array}{c}\sigma_{1} \\
\left(\mathrm{~km} \mathrm{~s}^{-1}\right) \\
\end{array}$ & $\begin{array}{c}O-C_{1} \\
\left(\mathrm{~km} \mathrm{~s}^{-1}\right) \\
\end{array}$ & $\begin{array}{c}\epsilon_{1} \\
\left(\mathrm{~km} \mathrm{~s}^{-1}\right) \\
\end{array}$ & $\begin{array}{c}\mathrm{RV}_{2} \\
\left(\mathrm{~km} \mathrm{~s}^{-1}\right) \\
\end{array}$ & $\begin{array}{c}\sigma_{2} \\
\left(\mathrm{~km} \mathrm{~s}^{-1}\right) \\
\end{array}$ & $\begin{array}{c}O-C_{2} \\
\left(\mathrm{~km} \mathrm{~s}^{-1}\right)\end{array}$ & $\epsilon_{2}$ & Inst. \\
\hline \multirow[t]{8}{*}{ HD78418 } & 53094.347119 & -14.83066 & 0.00854 & 0.00538 & 0.00299 & 38.51581 & 0.01243 & 0.00234 & 0.00580 & $\mathrm{HO}$ \\
\hline & $\begin{array}{c}53094.395761 \\
\ldots\end{array}$ & -14.91605 & 0.01039 & 0.00241 & 0.00663 & 38.60908 & 0.01267 & -0.00023 & 0.00628 & $\mathrm{HO}$ \\
\hline & 54101.570433 & -7.42109 & 0.00876 & -0.00731 & 0.00357 & 29.91678 & 0.01302 & 0.01759 & 0.00697 & $\mathrm{HN}$ \\
\hline & $\begin{array}{c}53744.479316 \\
\ldots\end{array}$ & 34.57326 & 0.00817 & 0.00352 & 0.00167 & -18.81645 & 0.01216 & 0.01278 & 0.00518 & $\mathrm{HN}$ \\
\hline & 54788.464774 & 18.30307 & 0.00696 & -0.00953 & 0.00485 & 0.16661 & 0.03029 & 0.01501 & 0.01374 & $\mathrm{H}$ \\
\hline & $\begin{array}{c}54430.494032 \\
\ldots\end{array}$ & -2.05150 & 0.00974 & -0.00216 & 0.00836 & 23.77923 & 0.03049 & 0.01071 & 0.01416 & $\mathrm{H}$ \\
\hline & 54190.964445 & 34.26932 & 0.01290 & 0.00397 & 0.01290 & -19.07279 & 0.01534 & 0.03793 & 0.01534 & $\mathrm{~S}$ \\
\hline & 54191.956955 & 29.78440 & 0.01249 & -0.01327 & 0.01249 & -13.94505 & 0.01050 & -0.01623 & 0.01050 & $S$ \\
\hline \multirow[t]{6}{*}{ HD123999 } & 53454.573974 & -51.41296 & 0.03155 & 0.00976 & 0.00585 & 72.66300 & 0.03675 & 0.00268 & 0.01119 & $\mathrm{HN}$ \\
\hline & $\begin{array}{c}53456.545316 \\
\ldots\end{array}$ & 39.94170 & 0.03231 & -0.02204 & 0.00911 & -21.65504 & 0.03623 & -0.04321 & 0.00937 & $\mathrm{HN}$ \\
\hline & 53978.858598 & 24.88006 & 0.04693 & 0.00628 & 0.03523 & -6.43652 & 0.03679 & -0.01637 & 0.03679 & S \\
\hline & $\begin{array}{c}54247.021370 \\
\ldots\end{array}$ & 48.74097 & 0.03697 & 0.07561 & 0.02015 & -31.00316 & 0.03535 & -0.04846 & 0.03535 & $\mathrm{~S}$ \\
\hline & 54107.560803 & -52.70193 & 0.02118 & -0.02634 & 0.01388 & 73.95953 & 0.03339 & -0.02783 & 0.01819 & $\mathrm{H}$ \\
\hline & 54108.572117 & -25.53836 & 0.04058 & -0.01830 & 0.03730 & 45.98819 & 0.05134 & 0.02694 & 0.04303 & $\mathrm{H}$ \\
\hline
\end{tabular}

Notes. $\sigma_{1,2}$ denote the total errors used in the least-squares fits and $\epsilon_{1,2}$ denote the internal errors. The last column denotes the spectrograph used to obtain a measurement. HN stands for Keck I/HIRES with the upgraded detector, HO for with the old detector, S stands for TNG/Sarg, and H for Shane/CAT/Hamspec.

(This table is available in its entirety in a machine-readable form in the online journal. A portion is shown here for guidance regarding its form and content.)

where $\Delta \lambda_{s}$ is the shift of the star spectrum, $\Delta \lambda_{\mathrm{I}_{2}}$ is the shift of the iodine transmission function $T, \otimes$ represents a convolution, and PSF a spectrograph's point-spread function (PSF). The parameters $\Delta \lambda_{s}, \Delta \lambda_{\mathrm{I}_{2}}$ as well as parameters describing the PSF are determined by performing a least-squares fit to the observed (through the iodine cell) spectrum $I$. For this purpose, one also needs (1) a high $\mathrm{S} / \mathrm{N}$ star spectrum taken without the cell, $F$ (the intrinsic stellar spectrum), which serves as a template for all the spectra observed through the cell and (2) the $\mathrm{I}_{2}$ transmission function $T$ obtained with a Fourier Transform Spectrometer such 
as the one at the Kitt Peak National Observatory. The Doppler shift of a star spectrum is then given by $\Delta \lambda=\Delta \lambda_{s}-\Delta \lambda_{\mathrm{I}_{2}}$. Such an iodine technique can only be applied to single stars. This is dictated by the need to supply an observed template spectrum of a star in Equation (1). In the case of SB2s, it cannot be accomplished since their spectra are always composite and time variable.

We can measure precise RVs of both components of an SB2 with an $\mathrm{I}_{2}$ absorption cell by performing the following steps. First, contrary to the standard approach for single stars, we always take two subsequent exposures of a binary target-one with and the other without the $I_{2}$ cell. This way we obtain an instantaneous template which is used to model only the adjacent exposure taken with the cell. Next, we perform the usual leastsquares fit and obtain the parameters described in Equation (1). Obviously, the derived Doppler shift, $\Delta \lambda_{i}$ (where $i$ denotes the epoch of the observation), carries no meaning since each time a different template is used (besides it describes a Doppler "shift" of a composite spectrum different at each epoch). However, the parameters (in particular the wavelength solution and the parameters describing PSF) are accurately determined and can be used to extract the star spectrum, $I^{\star, t}(\lambda)$, for each epoch $t$, by inverting Equation (1),

$$
I^{\star, t}(\lambda)=\left[I^{t}(\lambda) \otimes^{-1} \operatorname{PSF}^{t}\right] / T(\lambda),
$$

where $\otimes^{-1}$ denotes deconvolution and $\mathrm{PSF}^{t}$, symbolically, the set of parameters describing PSF at the epoch $t$. Such a star spectrum has an accurate wavelength solution, is free of the $\mathrm{I}_{2}$ lines and the influence of a varying PSF. In the final step, the velocities of both components of a binary target can be measured with the well-known two-dimensional crosscorrelation technique TODCOR (Zucker \& Mazeh 1994; Zucker et al. 2004) using as templates the synthetic spectra derived with the ATLAS 9 and ATLAS 12 programs (Kurucz 1995) and matched to the observed template spectrum, $F(\lambda)$.

\subsection{Iodine Cell Data Pipeline}

In our data pipeline, the reduction process involves the following procedures. An observed stellar spectrum, $I$, is a convolution of an intrinsic stellar spectrum, $F$, with a spectrographs' PSF, $\psi$. Such a convolution in a discrete form can be written as (Valenti et al. 1995; Endl et al. 2000)

$$
I_{i}=\sum_{j=i-r}^{i+r} F_{j} \psi_{i-j}, \quad i=1, \ldots, N
$$

where $N$ is the number of pixels in the analyzed spectrum and $r$ is the range of PSF such as $\psi_{l}=0$ for $|l|>r$. It is beneficial to work with an oversampled version of the above equation,

$$
I_{i}=\sum_{j=o i}^{o(i+1)-1}\left(\sum_{k=j-r}^{j+r} F_{k} \psi_{j-k}\right), \quad i=1, \ldots, N
$$

where $o$ is the oversampling factor. This equation can be rewritten into two other useful formulae

$$
I_{i}=\sum_{j=-r}^{r}\left(\sum_{k=0}^{o-1} F_{j+k+o i}\right) \psi_{-j}, \quad i=1, \ldots, N
$$

and

$$
I_{i}=\sum_{j=-r}^{r+o-1}\left(\sum_{k=-j}^{o-j-1} \psi_{k}\right) F_{j+o i}, \quad i=1, \ldots, N
$$

They constitute a set of equations which can be written in the form $\mathbf{I}=\mathbb{R} \mathbf{X}$ where $\mathbf{I}$ represents a vector of the observed spectrum $I_{i}, i=1, \ldots, N$, and $\mathbf{X}$ represents a vector of the PSF $\psi_{l}, l=-r, \ldots, r$, or of the intrinsic stellar spectrum $F_{k}, k=1, \ldots, o N+2 r$. The matrix $\mathbb{R}$ can easily be inferred from the appropriate sums above. By solving Equation (5) one can derive the PSF given the observed and intrinsic stellar spectrum, and by solving Equation (6) one can derive the intrinsic stellar spectrum given the PSF and the observed spectrum. The latter process is obviously a deconvolution. The remaining needed set of equations is one that allows us to clean a stellar spectrum observed through an iodine cell from the iodine lines as it is symbolically described in Equation (2). This can easily be achieved by noting that in such a case $F_{k}$ in Equation (6) has to be replaced by $F_{k} T_{k}$ where $T_{k}$ is a known transmission function of the iodine cell. In consequence, the set of equations $\mathbf{I}=\mathbb{R} \mathbf{X}$ is replaced by $\mathbf{I}=\mathbb{R} \mathbb{T} \mathbf{X}$ where $\mathbb{T}$ is a diagonal matrix with the diagonal elements equal to $T_{k}$ and then solved for $\mathbf{X}$ $\left(X_{k}=F_{k}, k=1, \ldots, o N+2 r\right)$. In our data pipeline, Equations (5) and (6) are solved with the maximum entropy method using a commercially available MEMSYS5 software. Our data pipeline provides state-of-the-art $1 \mathrm{~m} \mathrm{~s}^{-1}$ or better RV precision for single stars (see Figure 2).

In practice, the data reduction process is carried out as follows. Each observing night, a calibration exposure of a rapidly rotating B star or a quartz lamp is taken through an iodine cell. Such a spectrum in principle contains only the iodine lines and is used to determine the first wavelength solution and the first estimate of the PSF. This is done by taking $F=1$ in Equation (1) and involves solving Equation (5) for $\psi_{k}$ with $F_{k}=T_{k}$. The transmission function $T$ of the iodine cell as a function of wavelength is obviously known. An observing sequence for an SB2 involves taking a pair of exposures one with and one without the $\mathrm{I}_{2}$ cell-the instantaneous composite template. The PSF is deconvolved from the template to obtain an intrinsic template spectrum, $F_{k}$, using Equation (6). The template is assumed to have a wavelength solution from the calibration exposure. Such a template is then used to determine the PSF and the wavelength solution for the exposure of SB2s taken with the cell. This is done by using Equation (1) and involves solving Equation (5) where $F_{k}$ is replaced by $F_{k} T_{k}$. In the final step, the new PSF is used in Equation (6) to obtain a deconvolved and free of the $\mathrm{I}_{2}$ lines SB2 spectrum. This final spectrum is ready to be used with TODCOR as in Konacki (2005).

\subsection{Tomographic Disentangling of Composite Spectra}

The disentangling of the spectra of SB2s is a well-known problem to which several more or less restricted solutions exist and are described in a rich literature. We have decided to follow the tomographic approach to the disentangling problem by Bagnuolo \& Gies (1991) which we numerically formulate and solve within the framework of MEMSYS5. The idea of the tomographic disentangling is presented in Figure 3. Once the real template spectra for the components of a binary are available, it is no longer necessary to use TODCOR as one can simply use real templates and the original Equation (1) by replacing $F(\lambda+\Delta \lambda)$ with $S^{1}\left(\lambda+\Delta \lambda^{1}\right)+\delta_{S} S^{2}\left(\lambda+\Delta \lambda^{2}\right)$ where 

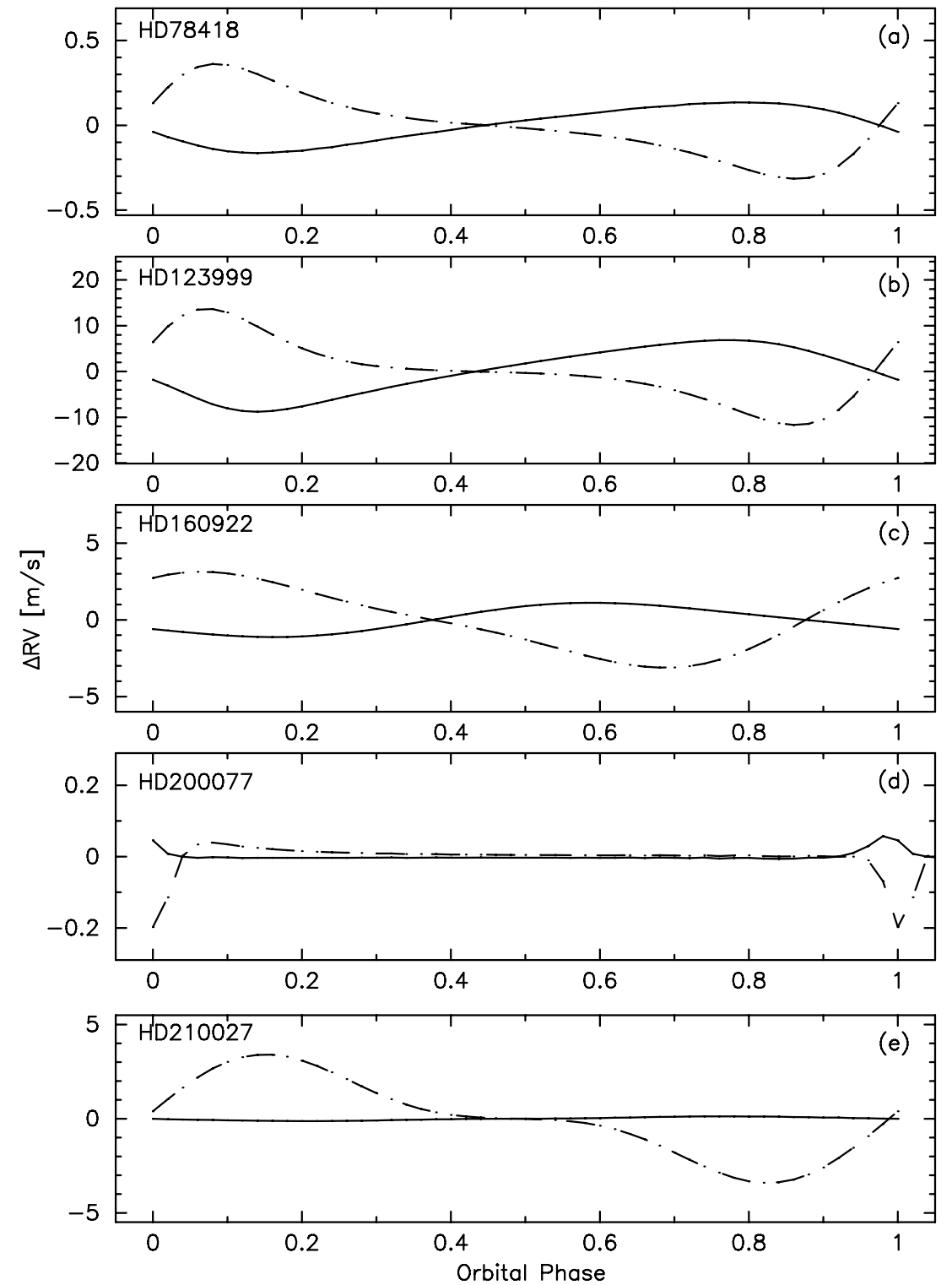

Figure 5. Radial velocity variations due to tidal distortion of the binary components as a function of the orbital phase for HD78418 (a), HD123999 (b), HD160922 (c), HD200077 (d), and HD210027 (e). The solid line is for the primary and the dash-dotted line for the secondary.

Table 2

Assumed Parameters for HD78418, HD123999, HD160922, HD200077, and HD210027

\begin{tabular}{|c|c|c|c|c|c|}
\hline Parameter & HD78418 & HD123999 & HD160922 & HD200077 & HD210027 \\
\hline Eff. temperature, primary, $T_{1}(\mathrm{~K})$ & 6000 & 6130 & 6500 & 6000 & 6642 \\
\hline Eff. temperature, secondary, $T_{2}(\mathrm{~K})$ & 5900 & 6230 & 5900 & 5500 & 4991 \\
\hline Potential, primary, $\Omega_{1}$ & 27.1 & 12.03 & 14.3 & 117.0 & 20.0 \\
\hline Potential, secondary, $\Omega_{2}$ & 34.9 & 15.21 & 14.1 & 114.0 & 22.5 \\
\hline Synchronization factor, primary, $F_{1}$ & 1.51 & 1.5 & 1.0 & 6.57 & 1.0 \\
\hline Synchronization factor, secondary, $F_{2}$ & 1.51 & 1.5 & 1.0 & 6.57 & 1.0 \\
\hline Gravity darkening exponent, primary, $g_{1}$ & 0.4 & 0.35 & 0.3 & 0.35 & 0.3 \\
\hline Gravity darkening exponent, secondary, $g_{2}$ & 0.4 & 0.35 & 0.3 & 0.4 & 0.4 \\
\hline Albedo, primary, $A_{1}$ & 0.5 & 0.5 & 0.5 & 0.5 & 0.5 \\
\hline Albedo, secondary, $A_{2}$ & 0.5 & 0.5 & 0.5 & 0.5 & 0.5 \\
\hline Metallicity & -0.09 & 0.0 & 0.0 & -0.14 & 0.0 \\
\hline Apparent diameter, primary, $\theta_{1}$ (mas) & 0.45 & 0.638 & 0.5 & 0.26 & 1.06 \\
\hline Apparent diameter, secondary, $\theta_{2}$ (mas) & 0.30 & 0.480 & 0.4 & 0.21 & 0.6 \\
\hline
\end{tabular}

Note.

a The more accurate parameters for HD123999 and HD210027 come from Boden et al. (2005) and Morel et al. (2000), respectively. The remaining ones are our best estimates based on the available information about the targets. 


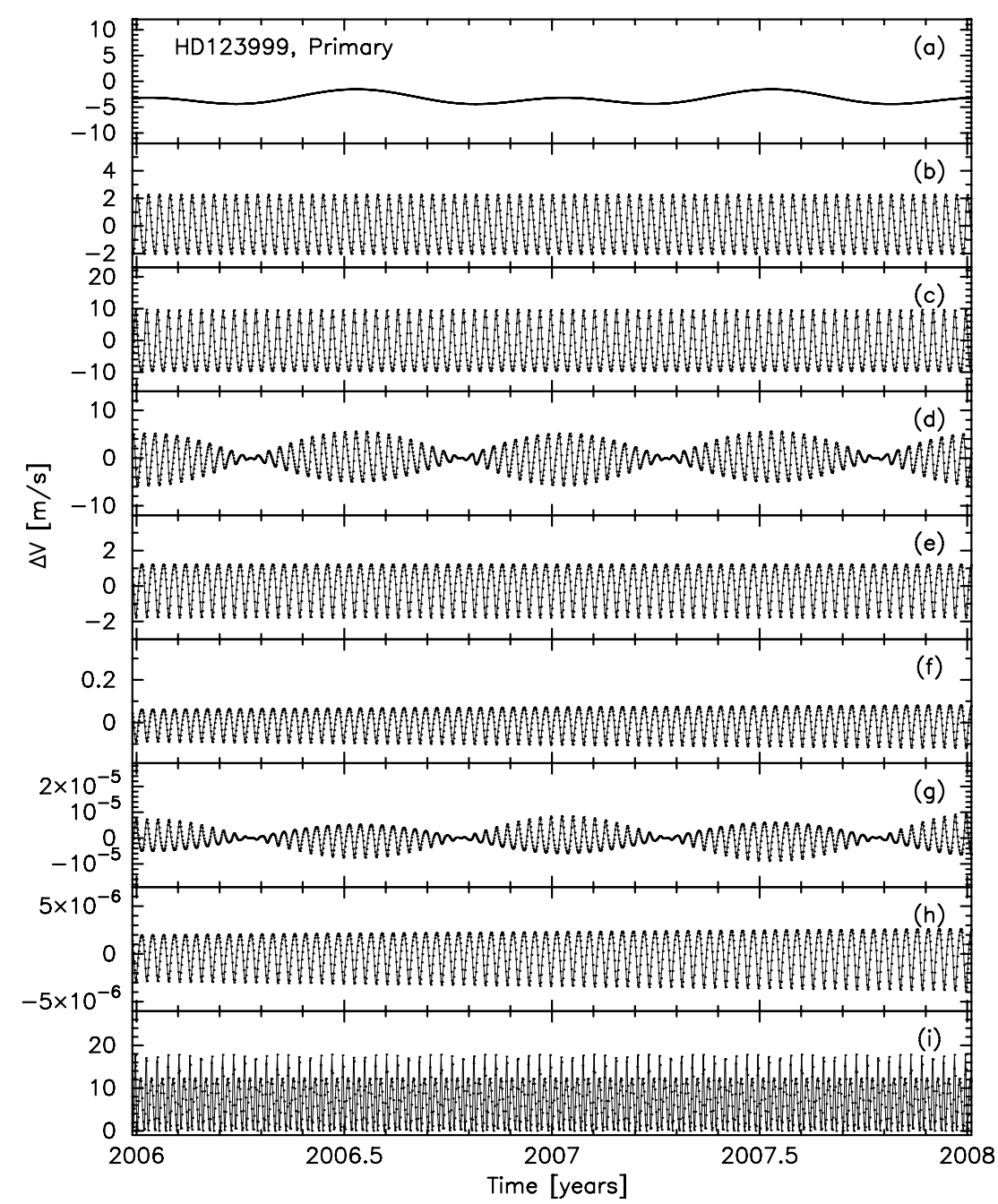

Figure 6. Radial velocity variations due to the relativistic effects $(a-h)$ and the light-time effect (i) for the primary star of HD123999. In the figure, (a) is for the term $\frac{1}{2 c}\left(V_{R}^{2}+V_{T}^{2}\right)-\frac{1}{c}\left(\frac{1}{2} V_{E}^{2}+U_{E}\right)-\frac{V_{R}}{c} \mathbf{S} \cdot \mathbf{V}_{G}-\frac{1}{c}\left(\frac{V_{G}^{2}}{2}+U_{\mathrm{SS}}-\left(\mathbf{S} \cdot \mathbf{V}_{G}\right)^{2}\right),(\mathrm{b})$ is for the term $\frac{1}{c} V_{R} \mathcal{V}_{z},(\mathrm{c})$ is for the term $\frac{1}{c}\left(\frac{1}{2} \mathcal{V}^{2}+U_{C}\right),(\mathrm{d})$ is for the term $-\frac{1}{c} \mathbf{S} \cdot \mathbf{V}_{G} \mathcal{V}_{z}$, (e) is for the term $\frac{1}{c}\left(\mathcal{V}_{x} V_{x}+\mathcal{V}_{y} V_{y}\right)$, (f) is for the term $\left(\mathcal{V}_{x} \mu_{\delta}+\mathcal{V}_{y} \mu_{\alpha}\right)\left(t-t_{0}\right)$, (g) is for the term $-\frac{1}{c} \mathbf{S} \cdot \mathbf{V}_{G}\left(\mathcal{V}_{x} \mu_{\delta}+\mathcal{V}_{y} \mu_{\alpha}\right)\left(t-t_{0}\right)$, and (h) is for the term $\frac{1}{c} V_{R}\left(\mathcal{V}_{x} \mu_{\delta}+\mathcal{V}_{y} \mu_{\alpha}\right)\left(t-t_{0}\right)$.

$S^{1}, S^{2}$ represent the two templates, $\Delta \lambda^{1}, \Delta \lambda^{2}$ their shifts, and $\delta_{S}$ the brightness ratio. Still TODCOR remains an important intermediate step to get the first approximate RVs that are subsequently used in the disentangling scheme.

It is worth noting that the composite spectra have to be continuum-normalized before they are tomographically disentangled and that the tomographic disentangling does not provide the real brightness ratio but only reproduces the depth of the spectral lines with respect to the normalized continuum of the composite spectrum. Hence, $\delta_{S}$ is not the actual brightness ratio. Successful tomographic disentangling can be carried out based on as little as $\sim 10$ spectra given that they sample different RVs of the components.

\subsection{RVs and Their Errors}

The spectra used to derive RVs were collected with four telescopes and three spectrographs, the Keck I/HIRES, Shane/ CAT/Hamspec, and TNG/Sarg, over the years 2003-2008. All three instruments are echelle spectrographs, which in our setup provide spectra with a resolution of $67,000,60,000$, and 86,000 , respectively, and are equipped with iodine cells. The observation was carried out as described above. The typical signal-to-noise ratios $(\mathrm{S} / \mathrm{Ns})$ per collapsed pixel of the collected spectra are $\sim 250$ for the Keck I/HIRES, $\sim 75-150$ for the TNG/Sarg, and $\sim 50-150$ for the Shane/CAT/Hamspec. These are for the composite observed spectra of SB2s. Hence, for example, a brightness ratio of 2.3 (HD78418) corresponds to an S/N of $\sim 175$ for the primary component and an $\mathrm{S} / \mathrm{N}$ of $\sim 75$ for the secondary. The RVs of the primaries are thus typically more accurate than of the secondaries. The formal RV errors were computed from the scatter between echelle orders. As it turns out these errors are underestimated. In order to obtain a reduced $\chi^{2}$ equal to 1 for a spectroscopic orbital solution and thus conservative estimates of the errors of the least-squares bestfit parameters, we add an additional error in quadrature to the formal errors (see Section 4). The most likely cause of the underestimation of the formal errors, in addition to the RV jitter of the stars, is subtle imperfections in the template component spectra obtained with the tomographic disentangling and used as a reference for RV computation. Figure 4 demonstrates how subtle the problem is. The sum of tomographically disentangled component spectra provides an overall better match to observed spectra than a single composite observed spectrum. Still in many 

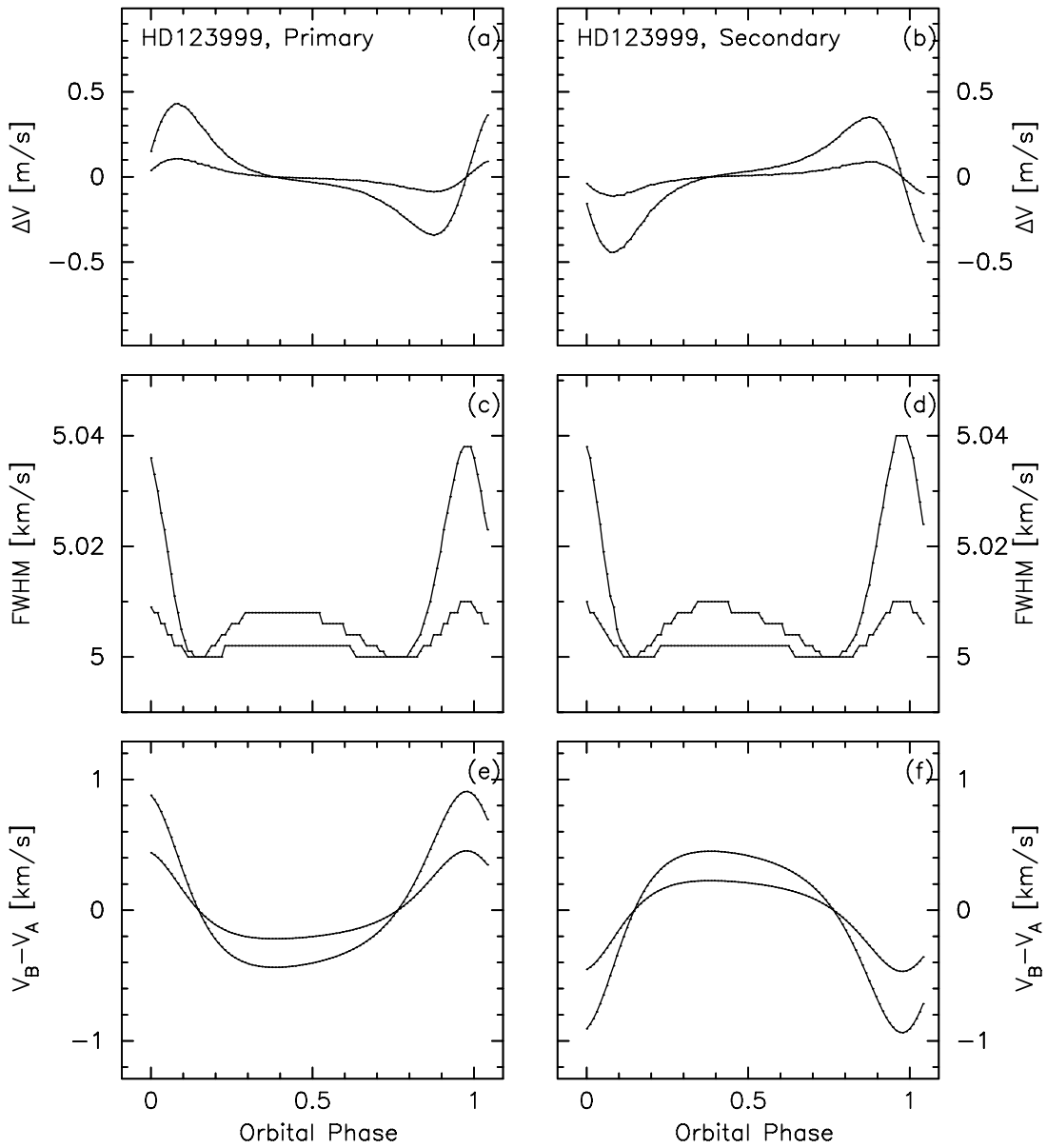

Figure 7. Impact of RV variations of the components of HD123999 over the duration of an exposure on the achievable RV precision. (a and b) the expected contribution to RVs. (c and d) the expected contribution to FWHM of the spectral lines (the edges of the lines are due to the finite sampling of $1 \mathrm{~m} \mathrm{~s}^{-1}$ ). (e and f) the difference between RVs of the components at the end and beginning of an exposure. In all the figures, the larger variations correspond to the integration time of $1200 \mathrm{~s}$ and the smaller ones to $600 \mathrm{~s}$.

Table 3

Calibration Stars for Visibility Measurements of HD78418, HD123999, HD160922, HD200077, and HD210027ª

\begin{tabular}{|c|c|c|c|c|c|}
\hline Target & Calibrator & $\begin{array}{l}\text { Spectral } \\
\text { Type }\end{array}$ & Magnitude & $\begin{array}{l}\text { Angular Separation } \\
\text { from Target (deg) }\end{array}$ & $\begin{array}{c}\text { Apparent } \\
\text { Diameter (mas) }\end{array}$ \\
\hline \multicolumn{6}{|l|}{ HD78418 } \\
\hline & HD79452 & G6III & $6.0 \mathrm{~V}, 3.8 \mathrm{~K}$ & 8.1 & 0.79 \\
\hline & HD73192 & K2III & $6.0 \mathrm{~V}, 3.3 \mathrm{~K}$ & 9.0 & 1.38 \\
\hline \multicolumn{6}{|c|}{ HD123999 } \\
\hline & HD121107 & G5III & $5.7 \mathrm{~V}, 3.6 \mathrm{~K}$ & 8.2 & 0.70 \\
\hline & HD128167 & F3V & $4.5 \mathrm{~V}, 3.6 \mathrm{~K}$ & 7.1 & 0.79 \\
\hline & HD123612 & K5III & $6.6 \mathrm{~V}, 3.0 \mathrm{~K}$ & 0.9 & 1.29 \\
\hline \multicolumn{6}{|c|}{ HD160922 } \\
\hline & HD154633 & G5V & $6.1 \mathrm{~V}, 4.5 \mathrm{~K}$ & 5.4 & 0.31 \\
\hline & HD158633 & $\mathrm{KOV}$ & $6.4 \mathrm{~V}, 4.4 \mathrm{~K}$ & 1.8 & 0.59 \\
\hline & HD168151 & F5V & $5.0 \mathrm{~V}, 3.9 \mathrm{~K}$ & 5.7 & 0.56 \\
\hline \multicolumn{6}{|c|}{ HD200077 } \\
\hline & HD192640 & $\mathrm{A} 2 \mathrm{~V}$ & $4.9 \mathrm{~V}, 4.9 \mathrm{~K}$ & 9.5 & 0.71 \\
\hline & HD192985 & F5V & $5.9 \mathrm{~V}, 4.8 \mathrm{~K}$ & 9.6 & 0.39 \\
\hline & HD199763 & G9III & $6.5 \mathrm{~V}, 4.3 \mathrm{~K}$ & 9.9 & 0.78 \\
\hline \multicolumn{6}{|c|}{ HD210027 } \\
\hline & HD211006 & K2III & $5.9 \mathrm{~V}, 3.2 \mathrm{~K}$ & 3.6 & 1.06 \\
\hline & HD211432 & G9III & $6.4 \mathrm{~V}, 4.2 \mathrm{~K}$ & 3.2 & 0.70 \\
\hline & HD215510 & G6III & $6.3 \mathrm{~V}, 4.2 \mathrm{~K}$ & 10.7 & 0.85 \\
\hline & HD210459 & F5III & $4.3 \mathrm{~V}, 2.5 \mathrm{~K}$ & 7.9 & 0.81 \\
\hline
\end{tabular}

Note.

${ }^{\text {a }}$ The adopted diameters of the calibrators are determined from their effective temperature and bolometric flux derived from archival photometry using getCal tool (ver. 2.10.7) supplied by the NASA Exoplanet Science Institute. 

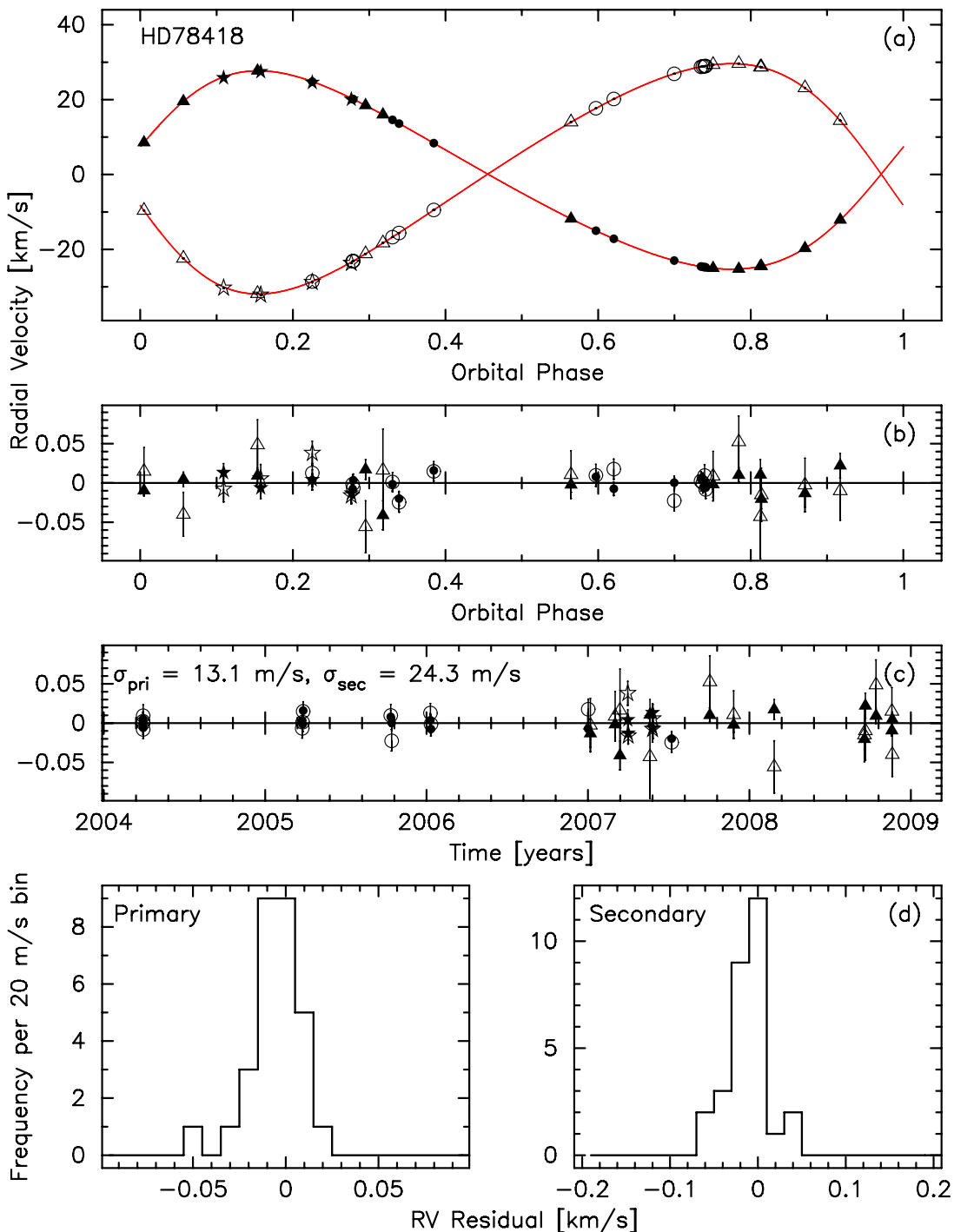

Figure 8. Observed and modeled radial velocities of HD78418 as a function of the orbital phase (a), their best-fit residuals as a function of the orbital phase (b) and time (c). The histograms of the residuals for the primary and secondary (d). The Keck I/HIRES is denoted with circles, Shane/CAT/Hamspec with triangles, and TNG/Sarg with stars.

(A color version of this figure is available in the online journal.)

Table 4

Best-fit Orbital Solutions for HD78418, HD123999, HD160922, HD200077, and HD210027

\begin{tabular}{|c|c|c|c|c|c|}
\hline Parameter & HD78418 & HD123999 & HD160922 & HD200077 & HD210027 \\
\hline Apparent semimajor axis, $\hat{a}$ (mas) & $5.8696(96)$ & $3.4706(55)$ & $3.469(17)$ & $14.453(18)$ & $10.329(16)$ \\
\hline Period, $P$ (days) & $19.412347(23)$ & $9.6045601(36)$ & $5.2797766(44)$ & $112.5132(13)$ & $10.2130253(16)$ \\
\hline Time of periastron, $T_{p}$ (TDB-2400000.5) & $53895.4025(24)$ & $54099.93572(70)$ & $54348.583(83)$ & $53830.169(14)$ & $52997.378(52)$ \\
\hline Eccentricity, $e$ & $0.19494(11)$ & $0.19214(15)$ & $0.00220(31)$ & $0.66227(51)$ & $0.001764(63)$ \\
\hline Longitude of the periastron, $\omega(\mathrm{deg})$ & $283.389(39)$ & $286.832(29)$ & $314.8(5.6)$ & 197.072(25) & $272.8(1.8)$ \\
\hline Longitude of the ascending node, $\Omega(\mathrm{deg})$ & $171.892(85)$ & $80.49(10)$ & $1.23(32)$ & $89.403(28)$ & $176.262(75)$ \\
\hline Inclination, $i(\mathrm{deg})$ & $146.88(25)$ & $107.95(12)$ & 151.4(1.1) & $118.682(80)$ & $95.83(12)$ \\
\hline Magnitude difference ( $K$ band), $\Delta K$ & $1.1445(131)$ & $0.601(13)$ & $0.841(18)$ & $1.1968(88)$ & $1.675(15)$ \\
\hline Magnitude difference ( $H$ band), $\Delta H$ & $1.1726(349)$ & $0.667(30)$ & $\ldots$ & $1.263(38)$ & $1.75(11)$ \\
\hline Velocity amplitude of the primary, $K_{1}\left(\mathrm{~km} \mathrm{~s}^{-1}\right)$ & 26.4961(35) & $67.189(11)$ & $36.254(16)$ & $29.373(82)$ & $48.4757(39)$ \\
\hline Velocity amplitude of the secondary, $K_{2}\left(\mathrm{~km} \mathrm{~s}^{-1}\right)$ & $30.7579(65)$ & $69.311(14)$ & $44.720(16)$ & $37.03(11)$ & $77.777(16)$ \\
\hline Gamma velocity, $V_{R}\left(\mathrm{~km} \mathrm{~s}^{-1}\right)$ & $9.7478(60)$ & $9.646(13)$ & $-14.011(19)$ & $-36.009(18)$ & $-4.6504(34)$ \\
\hline
\end{tabular}

Note.

${ }^{\mathrm{a}}$ The numbers in parentheses are the $1 \sigma$ errors in the last digits quoted. 

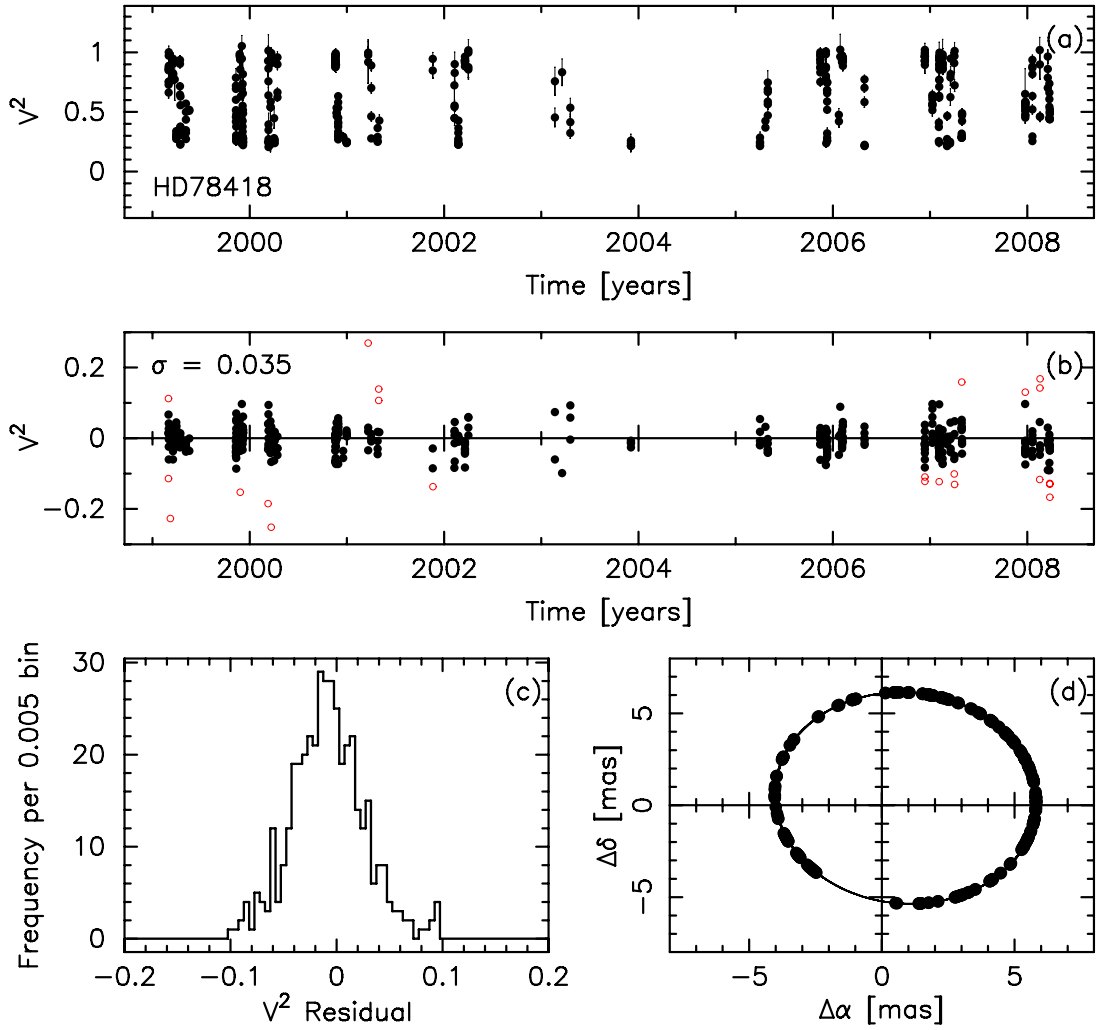

Figure 9. Visibility measurements of HD78418 as a function of time (a), their best-fit residuals as a function of time (b) and histogram (c). The measurements used to determine the best-fit orbital solution are denoted with filled circles. The corresponding orbital coverage and the relative orbit are shown in panel (d).

(A color version of this figure is available in the online journal.)

cases we do not yet reach a photon-limited RV precision for SB2s. This problem is being investigated and future releases of more accurate RVs from our program are very likely. The current RVs used in this paper are listed in Table 1.

\subsection{RV Modeling}

In a binary's center of mass coordinate system where the $x y$ plane is in the plane of the sky and the $z$-axis is directed away from the observer, the RVs may be modeled with the following equation:

$$
V_{\text {total }}=V_{R}+\mathcal{V}_{z}+\Delta V_{\text {tides }}+\Delta V_{\mathrm{SR} \mathrm{GR}}
$$

where $\mathcal{V}_{z}$ is a standard $\mathrm{RV}$ variation due to a Keplerian motion, $\Delta V_{\text {tides }}$ is an RV variation due to tidal distortion of the components, $\Delta V_{\mathrm{SR} \text { GR }}$ is a relativistic contribution to RVs, and $V_{R}$ is the $\mathrm{RV}$ of the center of mass of a binary (i.e., the gamma velocity).

It should be noted that the tidal, relativistic, and the light-time effects discussed below are subtle and the standard Keplerian model alone does a perfect job of taking them out. In consequence, when one attempts to fit for, e.g., the parameters related to the tidal effects together with the standard Keplerian parameters, one obtains an ill-conditioned least-squares problem. The main reason for including these effects is that they affect the best-fit values of the Keplerian parameters and hence the derived massess. This is an important issue when one claims a very high precision in masses. Yet, it is quite possible that with an even higher RV precision, one may be able to fit for some of the parameters related, e.g., to the tidal effects.

\subsubsection{Keplerian Motion and the Light-time Effect}

The standard Keplerian part of the RV variations is as follows:

$$
\mathcal{V}=\left(\mathcal{V}_{x}, \mathcal{V}_{y}, \mathcal{V}_{z}\right)=\frac{a n}{r}\left(-a \mathbf{P} \sin E+a \mathbf{Q} \sqrt{1-e^{2}} \cos E\right)
$$

$$
\begin{gathered}
n=2 \pi / P, \quad r=a(1-e \cos E), \quad \mathbf{P}=\mathbf{l} \cos \omega+\sin \omega, \\
\mathbf{Q}=-\mathbf{l} \sin \omega+\mathbf{m} \cos \omega, \quad \mathbf{l}=(\cos \Omega, \sin \Omega, 0), \\
\mathbf{m}=(-\cos i \sin \Omega, \cos i \cos \Omega, \sin i) .
\end{gathered}
$$

In particular, $\mathcal{V}_{z}$ can also be traditionally expressed as

$$
\begin{gathered}
\mathcal{V}_{z}=K(\cos (f+\omega)+e \cos \omega), \quad K=\frac{a n \sin i}{\sqrt{1-e^{2}}}, \\
\tan \frac{f}{2}=\sqrt{\frac{1+e}{1-e}} \tan \frac{E}{2},
\end{gathered}
$$

where $f$ is the true anomaly and $E=E(t)$ is the eccentric anomaly given by the Kepler equation $E-e \sin E=$ $2 \pi\left(t-T_{\mathrm{p}}\right) / P, P$ is the orbital period, $a, e, i, \omega, \Omega, T_{p}$ are the standard Keplerian elements - the semimajor axis of a component's orbit, the eccentricity, the inclination, the longitude of pericenter, the longitude of ascending node, and the time of pericenter. The spectroscopic orbits of the components can be fully described using the two respective RV amplitudes $K_{1,2}=a_{1,2} n \sin i / \sqrt{1-e^{2}}$, with the longitudes of pericenter satisfying the relation $\omega_{2}=\omega_{1}+\pi$ and the remaining orbital parameters being the same. Note that here $a_{1,2}$ refers to orbits of the components with respect to the center of mass of the binary. 

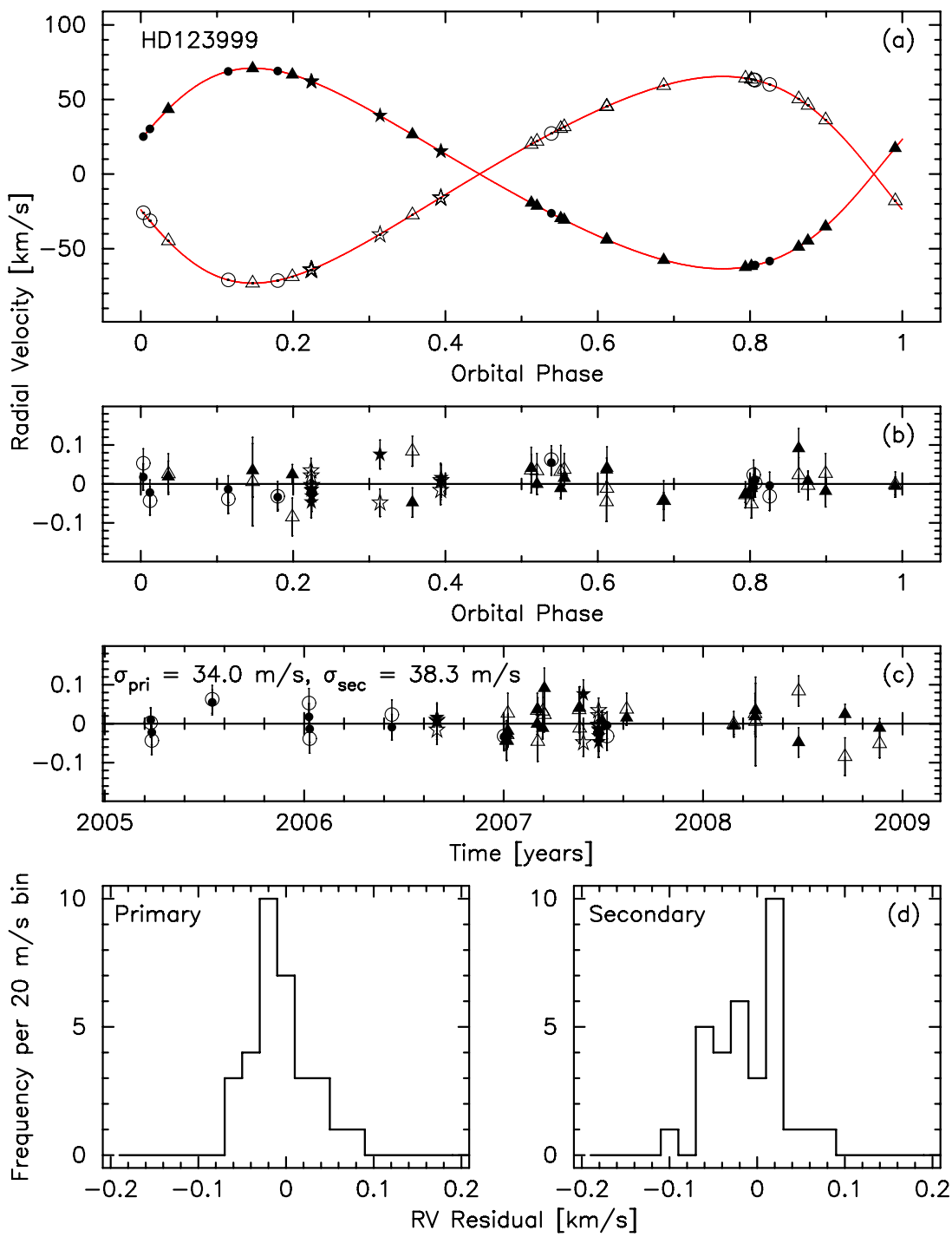

Figure 10. Observed and modeled radial velocities of HD123999 as a function of the orbital phase (a), their best-fit residuals as a function of the orbital phase (b) and time (c). The histograms of the residuals for the primary and secondary (d). The Keck I/HIRES is denoted with circles, Shane/CAT/Hamspec with triangles, and TNG/Sarg with stars.

(A color version of this figure is available in the online journal.)

At the level of precision $1-10 \mathrm{~m} \mathrm{~s}^{-1}$, it is important to include the light-time effect within the binary's orbit. We achieve this by solving the following implicit equation:

$$
\begin{aligned}
T\left(t_{1,2}\right) & =t_{1,2}+Z_{1,2}\left(t_{1,2}\right) / c, \\
Z_{1,2}\left(t_{1,2}\right) & =r \sin i \sin \left(f+\omega_{1,2}\right) \\
& =\frac{K_{1,2}}{c n(1+e \cos f)}\left(1-e^{2}\right)^{3 / 2} \sin \left(f+\omega_{1,2}\right),
\end{aligned}
$$

for $t_{1,2}$, where $T$ is the observed moment of an RV measurement referred to solar system barycenter (SSB; i.e., corrected for the light-time effect within the solar system), $t_{1,2}$ is the actual moment, and 1,2 refer to the primary and secondary, respectively. Subsequently, $t_{1,2}$ is used as the proper argument in the earlier equations. However, it is approximately true that

$$
t_{1,2} \approx T-Z_{1,2}\left(t_{1,2}\right) / c,
$$

and since $Z_{1,2}\left(t_{1,2}\right) / c$ is small, it can easily be derived that to the first order the RV contribution from the light-time effect is (see also Zucker \& Alexander 2007)

$$
\Delta V_{\mathrm{LTE}, 1,2}=\frac{1}{c} K_{1,2}^{2} \sin ^{2}\left(f+\omega_{1,2}\right)(1+e \cos f)
$$

and can be used to estimate the amplitudes of $\Delta V_{\mathrm{LTE}, 1,2}, K_{1,2}^{2} / c$, which are 2.9 and $3.2 \mathrm{~m} \mathrm{~s}^{-1}$ for HD78418, 15.1 and $16.0 \mathrm{~m} \mathrm{~s}^{-1}$ for HD123999, 4.4 and $6.7 \mathrm{~m} \mathrm{~s}^{-1}$ for HD160922, 2.9 and $4.6 \mathrm{~m} \mathrm{~s}^{-1}$ for HD200077, and 7.8 and $20.2 \mathrm{~m} \mathrm{~s}^{-1}$ for HD210027, for the primary and secondary, respectively.

\subsubsection{Tidal Effects}

The contributions of the tidally distorted binary components to the observed RVs were first noted by Sterne (1941). Such contributions arise from the non-spherical shapes of the binary's components and their non-uniform surface brightness due to predominantly gravitational and limb darkenings. The corresponding RV variations were analyzed by Wilson \& Sofia (1976) and Kopal (1980). Kopal (1980) made an attempt to derive an analytic approximation while Wilson \& Sofia (1976) used a more numerical approach employing the Wilson-Davinney 

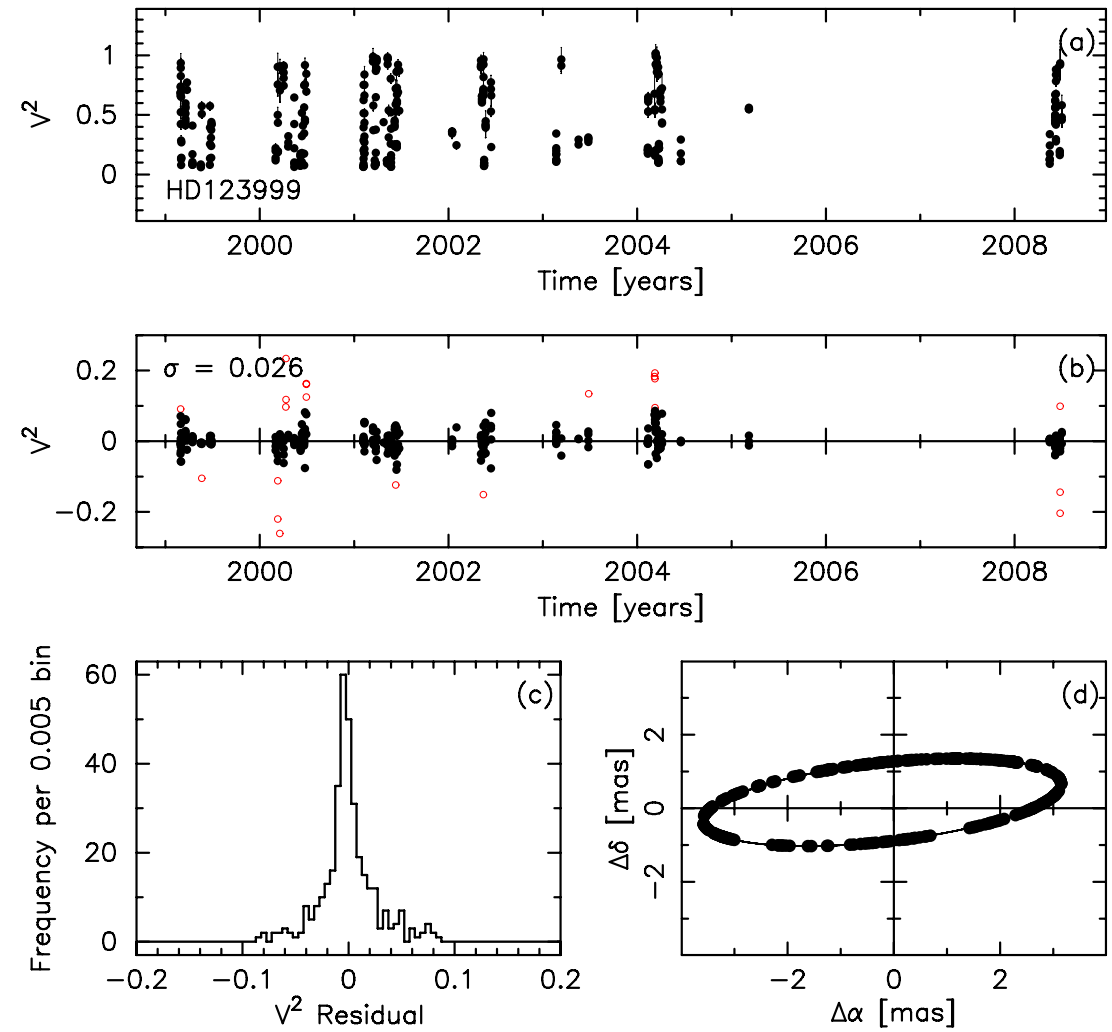

Figure 11. Visibility measurements of HD123999 as a function of time (a), their best-fit residuals as a function of time (b) and histogram (c). The measurements used to determine the best-fit orbital solution are denoted with filled circles. The corresponding orbital coverage and the relative orbit are shown in panel (d).

(A color version of this figure is available in the online journal.)

Table 5

Various Fit Parameters for HD78418, HD123999, HD160922, HD200077, and HD210027

\begin{tabular}{|c|c|c|c|c|c|}
\hline Parameter & HD78418 & HD123999 & HD160922 & HD200077 & HD210027 \\
\hline \multicolumn{6}{|l|}{ Velocity offsets } \\
\hline Secondary vs. primary $\left(\mathrm{km} \mathrm{s}^{-1}\right)$ & $0.239(12)$ & $0.032(21)$ & $0.116(29)$ & $0.343(42)$ & $0.333(16)$ \\
\hline HIRES old vs. new detector, primary, $v_{11}\left(\mathrm{~km} \mathrm{~s}^{-1}\right)$ & $-0.0009(71)$ & $\ldots$ & $\ldots$ & $\ldots$ & $-0.0053(38)$ \\
\hline HIRES old vs. new detector, secondary, $v_{12}\left(\mathrm{~km} \mathrm{~s}^{-1}\right)$ & $-0.0131(11)$ & $\ldots$ & $\cdots$ & $\cdots$ & $0.003(21)$ \\
\hline Hamspec vs. HIRES new detector, primary, $v_{21}\left(\mathrm{~km} \mathrm{~s}^{-1}\right)$ & $0.0358(67)$ & $-0.010(15)$ & $\cdots$ & $\cdots$ & $-0.0141(68)$ \\
\hline Hamspec vs. HIRES new detector, secondary, $v_{22}\left(\mathrm{~km} \mathrm{~s}^{-1}\right)$ & $0.054(12)$ & $0.042(18)$ & $\cdots$ & $\cdots$ & $0.026(27)$ \\
\hline Sarg vs. HIRES new detector, primary, $v_{31}\left(\mathrm{~km} \mathrm{~s}^{-1}\right)$ & $-0.3059(87)$ & $-0.182(24)$ & .. & $-0.3092(79)$ & $-0.3026(93)$ \\
\hline Sarg vs. HIRES new detector, secondary, $v_{32}\left(\mathrm{~km} \mathrm{~s}^{-1}\right)$ & $-0.286(10)$ & $-0.151(25)$ & $\cdots$ & $-0.228(23)$ & $-0.256(32)$ \\
\hline Hamspec vs. Sarg, primary, $v_{41}\left(\mathrm{~km} \mathrm{~s}^{-1}\right)$ & $\ldots$ & $\ldots$ & $0.262(21)$ & $\ldots$ & $\ldots$ \\
\hline Hamspec vs. Sarg, secondary, $v_{42}\left(\mathrm{~km} \mathrm{~s}^{-1}\right)$ & $\cdots$ & $\cdots$ & $0.344(21)$ & $\cdots$ & $\cdots$ \\
\hline \multicolumn{6}{|l|}{ Least-squares fit parameters } \\
\hline Number of RV measurements, total & 58 & 64 & 36 & 26 & 144 \\
\hline Number of RV measurements, Keck/HIRES & 26 & 16 & $\ldots$ & 18 & 102 \\
\hline Number of RV measurements, Shane/CAT/Hamspec & 24 & 34 & 20 & & 30 \\
\hline Number of RV measurements, TNG/Sarg & 8 & 14 & 16 & 8 & 12 \\
\hline Number of $V^{2}$ measurements & 415 & 346 & 61 & 329 & 266 \\
\hline Additional RV error, Keck/HIRES, primary/secondary $\left(\mathrm{m} \mathrm{s}^{-1}\right)$ & $8.0 / 11.0$ & $31.0 / 35.0$ & $\ldots$ & $5.0 / 26.0$ & $10.5 / 65.0$ \\
\hline Additional RV error, Shane/CAT/Hamspec, primary/secondary $\left(\mathrm{m} \mathrm{s}^{-1}\right)$ & $5.0 / 27.0$ & $16.0 / 28.0$ & $27.0 / 15.0$ & $\ldots$ & $18.5 / 68.0$ \\
\hline Additional RV error, TNG/Sarg, primary/secondary $\left(\mathrm{m} \mathrm{s}^{-1}\right)$ & $0.0 / 0.0$ & $31.0 / 0.0$ & $37.0 / 25.0$ & $0.0 / 26.0$ & $10.5 / 38.0$ \\
\hline Combined RV rms, primary/secondary $\left(\mathrm{m} \mathrm{s}^{-1}\right)$ & $13.1 / 24.3$ & $34.0 / 38.3$ & $37.9 / 31.9$ & $8.5 / 30.2$ & $17.1 / 69.7$ \\
\hline Keck/HIRES RV rms, primary/secondary $\left(\mathrm{m} \mathrm{s}^{-1}\right)$ & $6.9 / 14.3$ & $30.2 / 35.8$ & $\ldots$ & $6.7 / 17.1$ & $14.1 / 64.6$ \\
\hline Shane/CAT/Hamspec RV rms, primary/secondary $\left(\mathrm{m} \mathrm{s}^{-1}\right)$ & $18.2 / 33.8$ & $36.3 / 37.6$ & $25.6 / 14.8$ & $\ldots$ & $22.7 / 84.2$ \\
\hline TNG/Sarg RV rms, primary/secondary $\left(\mathrm{m} \mathrm{s}^{-1}\right)$ & $8.2 / 17.4$ & $40.2 / 26.8$ & $35.9 / 37.1$ & $10.4 / 25.9$ & $12.3 / 55.8$ \\
\hline$V^{2} \mathrm{rms}$ & 0.0351 & 0.0261 & 0.0274 & 0.0381 & 0.0281 \\
\hline $\mathrm{RV} \chi^{2}$, primary/secondary & $31.60 / 32.99$ & $28.21 / 28.20$ & $19.31 / 18.19$ & $11.27 / 13.24$ & $70.40 / 64.94$ \\
\hline$V^{2} \chi^{2}$ & 433.71 & 482.46 & 50.59 & 522.03 & 248.29 \\
\hline Degrees of freedom, dof & 454 & 393 & 83 & 355 & 391 \\
\hline Total reduced $\chi^{2}, \chi^{2} /$ dof & 1.096 & 1.371 & 1.061 & 1.607 & 0.981 \\
\hline
\end{tabular}

Note.

${ }^{\text {a }}$ The numbers in parentheses are the $1 \sigma$ errors in the last digits quoted. 

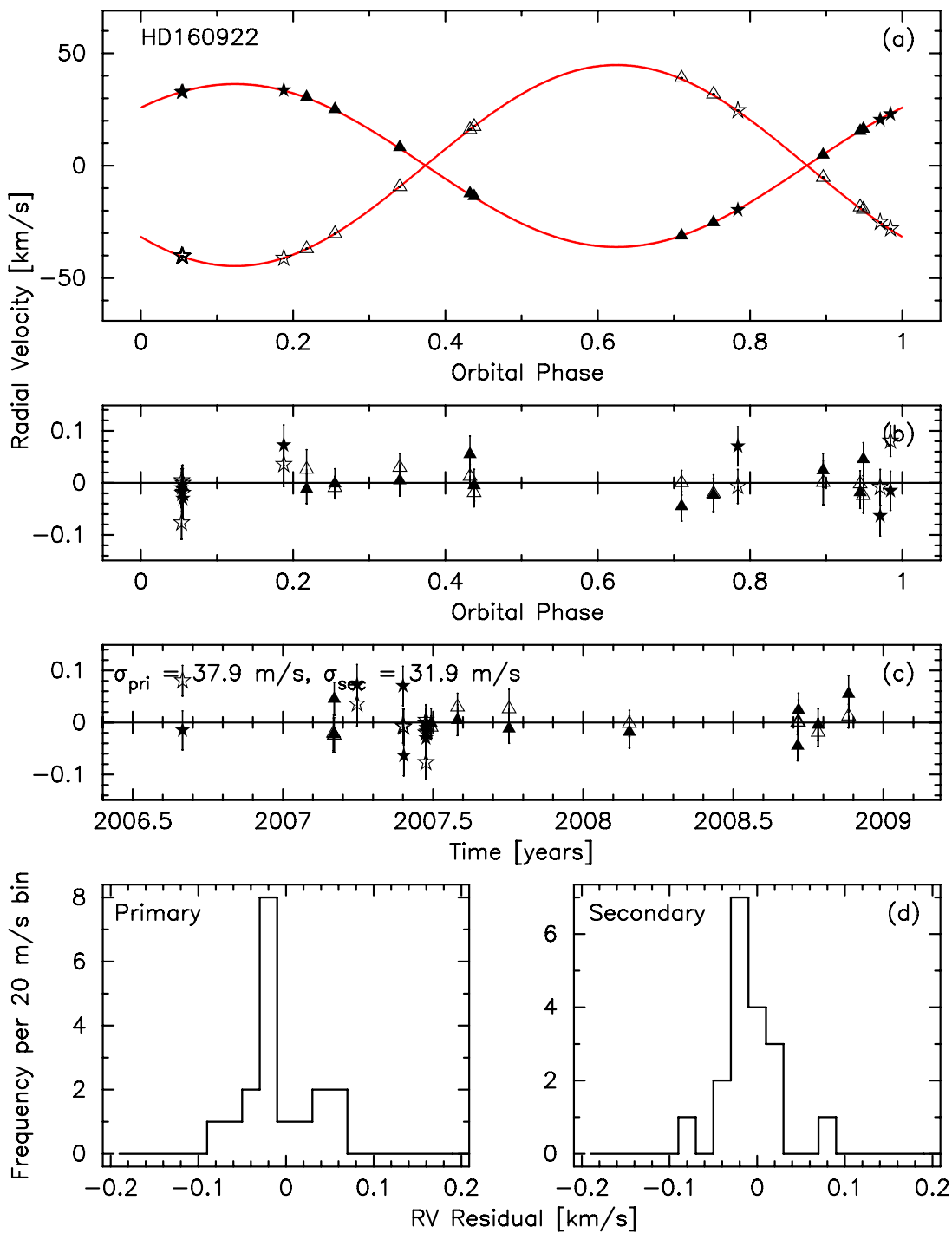

Figure 12. Observed and modeled radial velocities of HD160922 as a function of the orbital phase (a), their best-fit residuals as a function of the orbital phase (b) and time (c). The histograms of the residuals for the primary and secondary (d). The Keck I/HIRES is denoted with circles, Shane/CAT/Hamspec with triangles, and TNG/Sarg with stars.

(A color version of this figure is available in the online journal.)

code (Wilson \& Devinney 1971; Wilson 1979) for modeling eclipsing binary light curves to compute the "tidal" RV contribution. Recently, this effect was also analyzed by Eaton (2008). We follow the approach by Wilson \& Sofia (1976) and Eaton (2008) where the "tidal" contribution to the RVs is modeled with the Wilson-Davinney code. To this end, we use the light curve modeling program $\mathrm{l} \mathrm{c}$ which is part of the Wilson-Daviney code assuming the parameters of our targets as in Table 2. The resulting RV variations are shown in Figure 5. It should be noted that even though these effects are quite small they can have a significant qualitative effect if ignored by, e.g., mimicking a small orbital eccentricity in an otherwise circular orbit.

\subsubsection{Relativistic Effects}

The relativistic description of the motion of a binary star from the point of view of its RVs was derived by Kopeikin \& Ozernoy (1999). In our model, we adopt the following relativistic correction based on Equations (76)-(86) from Kopeikin \&
Ozernoy (1999):

$$
\begin{aligned}
\Delta V_{\mathrm{SR} \mathrm{GR}}= & \frac{1}{2 c}\left(V_{R}^{2}+V_{T}^{2}\right)-\frac{1}{c}\left(\frac{1}{2} V_{E}^{2}+U_{E}\right)-\frac{V_{R}}{c} \mathbf{S} \cdot \mathbf{V}_{G} \\
& -\frac{1}{c}\left(\frac{V_{G}^{2}}{2}+U_{\mathrm{SS}}-\left(\mathbf{S} \cdot \mathbf{V}_{G}\right)^{2}\right)-\frac{1}{c} \mathbf{S} \cdot \mathbf{V}_{G} \mathcal{V}_{z} \\
& -\frac{1}{c} \mathbf{S} \cdot \mathbf{V}_{G}\left(\mathcal{V}_{x} \mu_{\delta}+\mathcal{V}_{y} \mu_{\alpha}\right)\left(t-t_{0}\right) \\
& +\left(\mathcal{V}_{x} \mu_{\delta}+\mathcal{V}_{y} \mu_{\alpha}\right)\left(t-t_{0}\right)+\frac{1}{c} V_{R} \mathcal{V}_{z} \\
& +\frac{1}{c} V_{R}\left(\mathcal{V}_{x} \mu_{\delta}+\mathcal{V}_{y} \mu_{\alpha}\right)\left(t-t_{0}\right)+\frac{1}{c}\left(\mathcal{V}_{x} V_{x}+\mathcal{V}_{y} V_{y}\right) \\
& +\frac{1}{c}\left(\frac{1}{2} \mathcal{V}^{2}+U_{C}\right),
\end{aligned}
$$

where $V_{R}, V_{T}$ are the radial and tangential velocities of the center of mass of the binary, $V_{E}, U_{E}$ are the velocity magnitude and gravitational potential of the observer with respect to the 

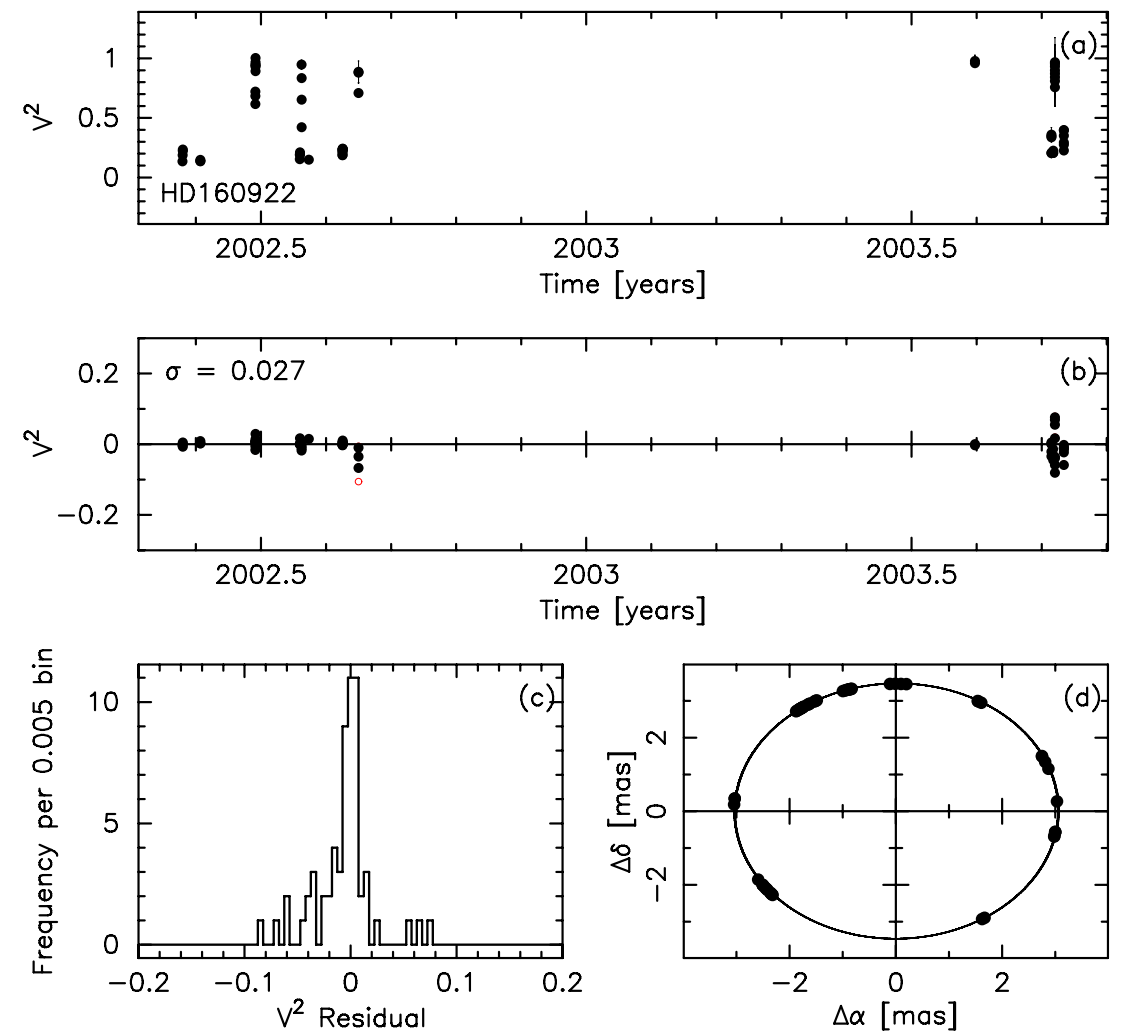

Figure 13. Visibility measurements of HD160922 as a function of time (a), their best-fit residuals as a function of time (b) and histogram (c). The measurements used to determine the best-fit orbital solution are denoted with filled circles. The corresponding orbital coverage and the relative orbit are shown in panel (d).

(A color version of this figure is available in the online journal.)

geocenter, $\mathbf{V}_{G}, V_{G}$ are the velocity vector and its magnitude of the geocenter with respect to the SSB, $U_{\mathrm{SS}}$ is the gravitational potential at the geocenter due to all major bodies in the solar system, $\mu_{\alpha}, \mu_{\delta}$ is the proper motion of the center of mass of the binary expressed in radians per time unit, $V_{x}, V_{x}$ are the components of the tangential velocity vector such as $V_{x}=p c \mu_{\alpha} / \kappa, V_{y}=p c \mu_{\delta} / \kappa$ where $\kappa$ is the parallax, $p c$ is $1 \mathrm{pc}$, and $\mathbf{S}$ is the unit vector toward the center of mass of the binary. $\mathcal{V}_{x}, \mathcal{V}_{y}, \mathcal{V}_{z}$ as before denote the coordinates of the orbital velocity vector of a given component, $\mathcal{V}$ is its magnitude, and $U_{C}$ is the gravitational potential at the position of a binary component due to the gravitational field of its companion. Note that $\mathbf{S}$ varies slowly due to the proper motion vector $\boldsymbol{\mu}$, $\mathbf{S}=\mathbf{S}+\boldsymbol{\mu}\left(t-t_{0}\right)$ and the term $\left(\mathcal{V}_{x} \mu_{\delta}+\mathcal{V}_{y} \mu_{\alpha}\right)\left(t-t_{0}\right)$ is not really relativistic but is obviously due to the varying $\mathbf{S}$. The necessary quantities, such as $\mathbf{V}_{G}, U_{E}, U_{\mathrm{SS}}, V_{T}, \mathbf{S}$, we calculated using the JPL ephemerides DE405 and the catalog positions and proper motions of the targets with the help of the NOVAS library of astrometric subroutines by Kaplan et al. (1989).

The last term in the above equation is the combined effect of the transverse Doppler effect and the gravitational redshift and also the dominant term in the relativistic correction. It can easily be shown that its periodic part has the following form:

$$
\begin{aligned}
\left(\frac{1}{2} \mathcal{V}^{2}+U_{C}\right)_{\text {periodic }} & =\gamma_{1,2} \cos f, \\
\gamma_{1,2} & =\frac{G M_{2,1}\left(M_{1,2}+2 M_{2,1}\right)}{c a\left(M_{1}+M_{2}\right)} \frac{e}{\left(1-e^{2}\right)}
\end{aligned}
$$

for the primary and secondary, respectively, where $M_{1}, M_{2}$ are the masses of the components. One can employ the above equation to test general relativity by using $\gamma_{1,2}$ as a free parameter and fitting for it. However, as noted by Kopeikin \& Ozernoy (1999), it is difficult in practice due to the coupling of this effect with the Keplerian part of the model. The above equation can be rewritten in the following form:

$$
\gamma_{1,2}=\frac{K_{1,2}\left(2 K_{1,2}+K_{2,1}\right)}{c \sin ^{2} i} e,
$$

and, as has been recently noted by Zucker \& Alexander (2007), can in principle be used to derive the orbital inclination using just the RV measurements. In particular, Zucker \& Alexander (2007) propose to use HD123999 to this end (see Figure 6).

Three of our targets HD78418, HD123999, and HD200077 have significant eccentricities and the resulting $\gamma_{1,2}$ are as follows: 4.8 and $5.9 \mathrm{~m} \mathrm{~s}^{-1}, 9.7$ and $10.1 \mathrm{~m} \mathrm{~s}^{-1}$, and 8.1 and $11.0 \mathrm{~m} \mathrm{~s}^{-1}$ for the primary and secondary, respectively. Only for HD78418, the procedure for deriving $\sin ^{2} i$ proposed by Zucker $\&$ Alexander (2007) provided a value of $0.3832(i=141.8$ $\mathrm{deg})$ close to the real one of $0.2985(i=146.9)$ as determined with the help of PTI astrometric data. However, the error of $\sin ^{2} i=0.3832$ is 1.8 , so this is more of a coincidence. The method may yet turn out to be useful given sufficiently accurate and/or numerous RV data sets.

Finally, let us note that our attempts to fit for $\dot{\omega}$, which could be either due to the relativistic or tidal precession (Mazeh 2008), have not produced any meaningful results. In four cases, $\dot{\omega}$ was at the level of its formal error. In one case (HD78418), $\dot{\omega}$ was technically relatively significant $\left((2.4 \pm 0.6) \times 10^{-4}\right.$ deg day $\left.^{-1}\right)$ but its inclusion in the model has not improved the best-fit rms. Hence, the orbital part of the description of the motion remains 

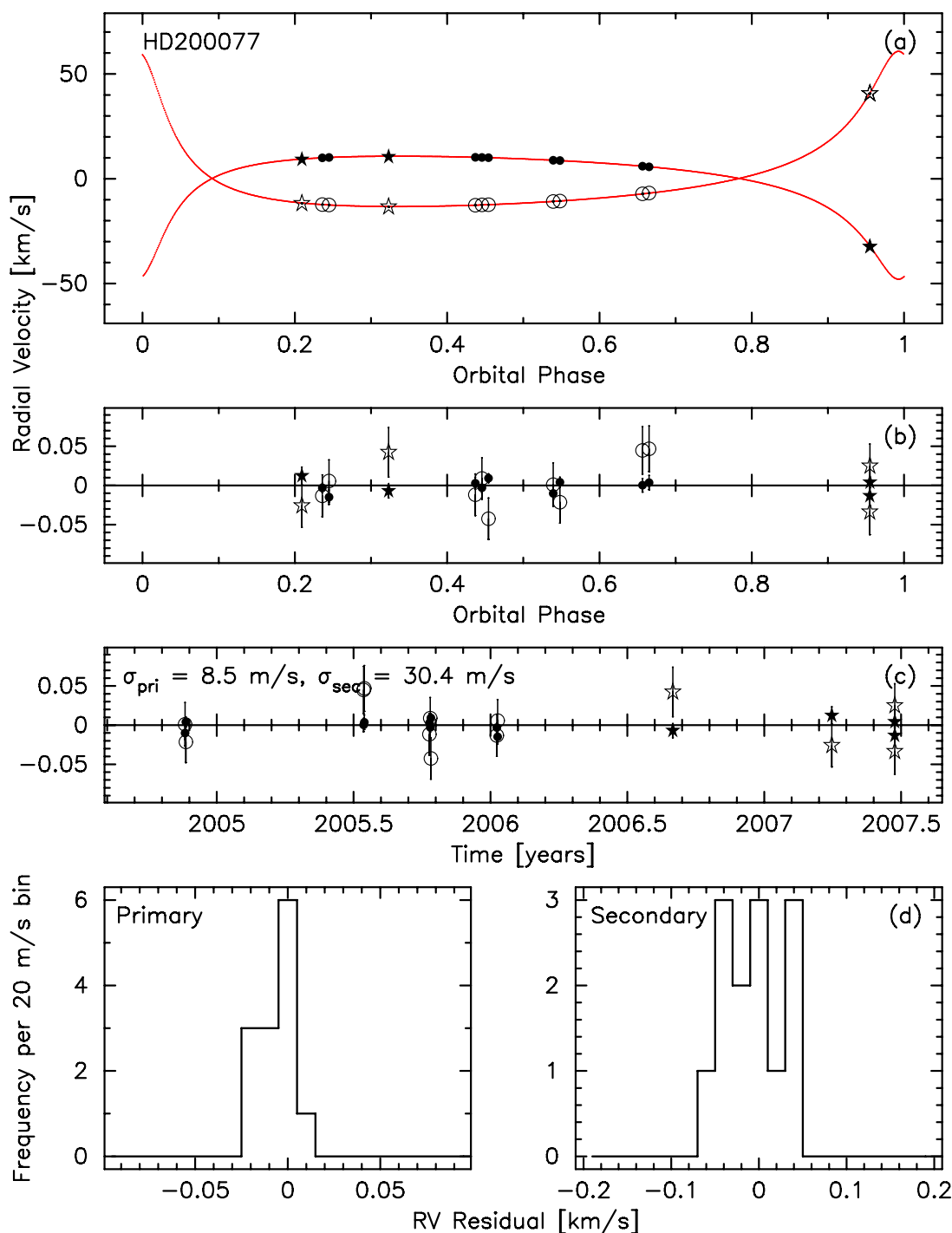

Figure 14. Observed and modeled radial velocities of HD200077 as a function of the orbital phase (a), their best-fit residuals as a function of the orbital phase (b) and time (c). The histograms of the residuals for the primary and secondary (d). The Keck I/HIRES is denoted with circles, Shane/CAT/Hamspec with triangles, and TNG/Sarg with stars.

(A color version of this figure is available in the online journal.)

in its classic Newtonian form as in Equation (8) throughout this paper.

\subsubsection{Exposure Times and Their Impact on RV Precision}

One of the aspects that differs single and binary stars is quite a significant RV variation of the components of a binary due to their orbital motion during an exposure. In order to explore if such an RV variation may impact the achievable RV precision, we proceeded as follows. For a given integration time, $T$, we computed a corresponding average $\mathrm{RV}, \hat{v}(t)$,

$$
\hat{v}(t)=\frac{1}{T} \int_{t-T / 2}^{t+T / 2} V(s) d s=(Z(t+T / 2)-Z(t-T / 2)) / T,
$$

where $t$ is the mid-point of an exposure, $V(t)$ is the RV, and $Z(t)$ is the Zth coordinate of a component in its Keplerian motion. It was then compared with an RV corresponding to the mid-point of an exposure, $V(t)$, as $\Delta V(t)=V(t)-\hat{v}$. Additionally, we also explored the influence of the varying RV on the width of the spectral lines by co-adding a series of Gaussian functions with a full width at half-maximum (FWHM) of $5 \mathrm{~km} \mathrm{~s}^{-1}$, approximately the narrowest spectral lines that we measured in our program, over the duration of an exposure. An example of such an analysis for HD123999 and the integration times of 600 and $1200 \mathrm{~s}$ is shown in Figure 7.

As can be seen the resulting RV variations, $\Delta V(t)$, are well below $1 \mathrm{~m} \mathrm{~s}^{-1}$. They rapidly diminish as the integration times become smaller than $600 \mathrm{~s}$. In our program, the typical integration times were the following: in the case of HD123999 6-42, 300, and 60-300 s, HD210027 3-40, 30-40, and 30-60 s, and HD78418 16-100, 190, and 100-150 s for the Keck/HIRES, Lick/Hamspec, and TNG/Sarg spectra, respectively; in the case of HD200077 20-180 and 220-180 s for the Keck/HIRES and TNG/Sarg and HD160922 120-180 and 70-300 s for the Lick/Hamspec and TNG/Sarg. Also the corresponding small variations in the shape (FWHM) of the spectral lines are not an issue as they are smaller than the typical variations in PSFs of a spectrograph. It should be remembered that contrary to spectrographs such as, e.g., HARPS, spectrographs employing 

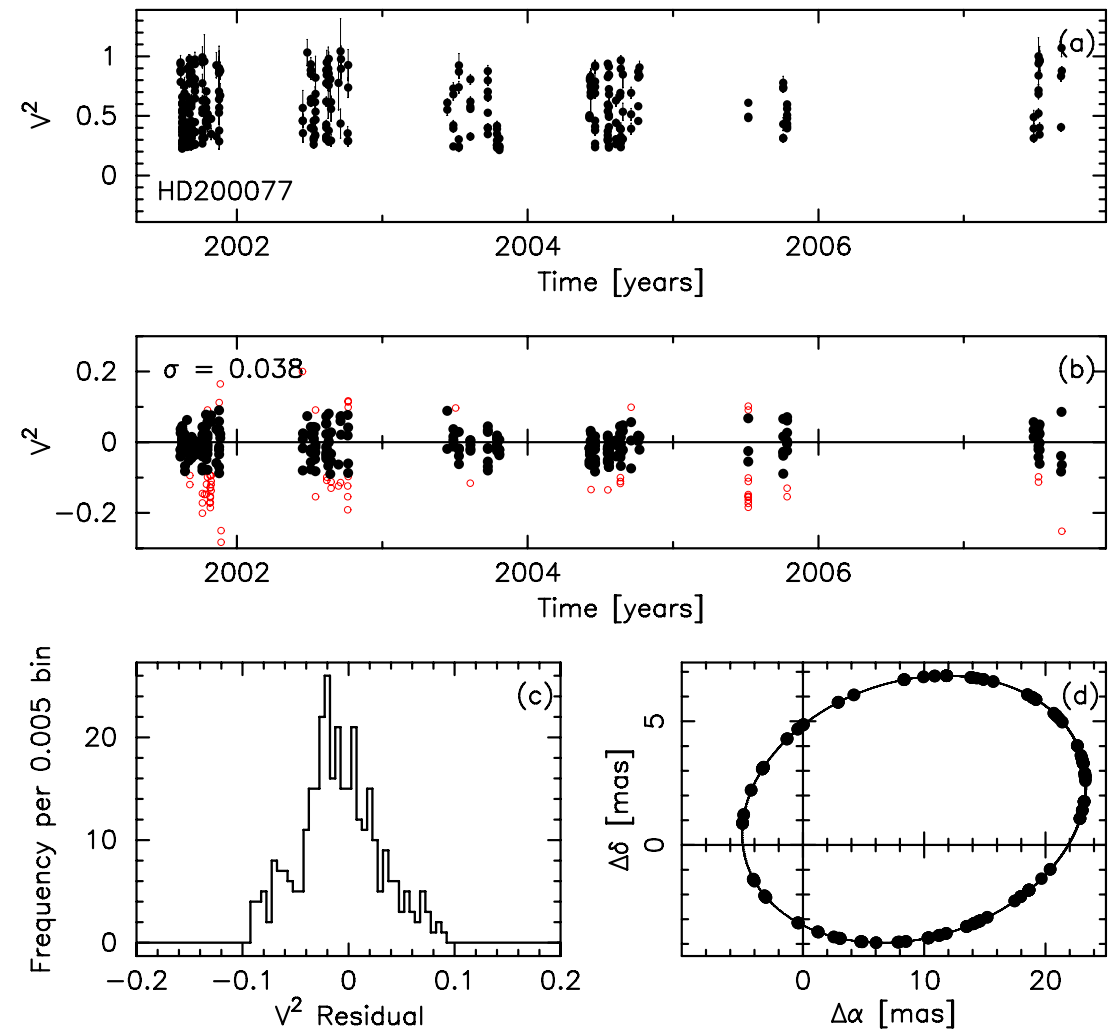

Figure 15. Visibility measurements of HD200077 as a function of time (a), their best-fit residuals as a function of time (b) and histogram (c). The measurements used to determine the best-fit orbital solution are denoted with filled circles. The corresponding orbital coverage and the relative orbit are shown in panel (d).

(A color version of this figure is available in the online journal.)

iodine cells are not nearly as stable. For example, the zero point of a wavelength solution of HIRES changes by several $\mathrm{km} \mathrm{s}^{-1}$ over a night (see, e.g., Konacki et al. 2003). Hence, among others the necessity is to model PSFs from exposure to exposure.

\section{VISIBILITIES AND THEIR MODELING}

Interferometers such as PTI typically measure a fringe contrast - a normalized (by the total power received from a source) amplitude of the coherence function. The normalized visibility of a binary star can be modeled with the following equation (see Boden 2000; Konacki \& Lane 2004):

$$
V_{\text {binary }}^{2}=\frac{V_{1}^{2}+r^{2} V_{2}^{2}+2 r V_{1} V_{2} \cos \left(2 \pi \mathbf{B}_{\perp} \cdot \Delta \mathbf{s} / \lambda\right)}{(1+r)^{2}}
$$

where $V_{1}, V_{2}$ are the visibilities of the components approximated with the visibility of a uniform disk of diameter $\theta$,

$$
V_{i}^{2}=\left(\frac{2 J_{1}\left(\pi \theta B_{\perp} \lambda\right)}{\pi \theta B_{\perp} \lambda}\right)^{2}, i=1,2,
$$

where $B_{\perp}=\left\|\mathbf{B}_{\perp}\right\|$ is the length of the projected baseline vector of a two-aperture interferometer, $r$ is the brightness ratio at the observing wavelength $\lambda\left(r=P_{2} / P_{1}\right.$ where $P_{1}, P_{2}$ are the total powers of the binary components at the given wavelength), and $\Delta \mathbf{s}=(\Delta \delta, \Delta \alpha)$ is the separation vector between the primary and the secondary in the plane tangent to the sky.

The separation vector between the primary and the secondary is given by the following equations (see, e.g., Kovalevsky 1995; van de Kamp 1967):

$$
\begin{aligned}
\Delta \mathbf{s}(t) & =(\Delta \delta(t), \Delta \alpha(t)) \\
& =\kappa a\left(\mathbf{P}(\cos E(t)-e)+\mathbf{Q} \sqrt{1-e^{2}} \sin E(t)\right),
\end{aligned}
$$

where $a$ is the semimajor axis of the relative orbit and the first two coordinates of the vectors $\mathbf{P}$ and $\mathbf{Q}$ are used. Note that traditionally the north direction is the $x$-axis and the east direction is the $y$-axis for a coordinate system used in astrometry to model the orbital motion. In our figures showing the relative orbits, the $x$-axis is for the right ascension and the $y$-axis for the declination but the orbital motion is modeled as above. Note also that a relative astrometric orbit allows for a few possible solutions for the pair of angles $\omega, \Omega$ namely, $\omega, \Omega$ as well as $\omega \pm \pi, \Omega \pm \pi$. The inclusion of a spectroscopic orbit allows one to determine the real $\omega$ and traditionally $\Omega$ is chosen to be less than $\pi$.

PTI provides a square of the normalized visibility amplitude, $V^{2}$, as one can obtain its unbiased estimate (Colavita 1999; Mozurkewich et al. 1991). A normalized visibility of a point source should be by definition equal to 1 . Since a real instrument is not perfect and does not operate in a perfect environment, this is typically not the case and the observed visibility is underestimated. In consequence, each visibility measurement has to be calibrated. This is carried out by observing at least one calibrator in between the observations of a target. The calibrator is typically a single star whose diameter is known and its visibility can be very well approximated with Equation (17). Then, the calibrated visibility of a target is given by (Boden 

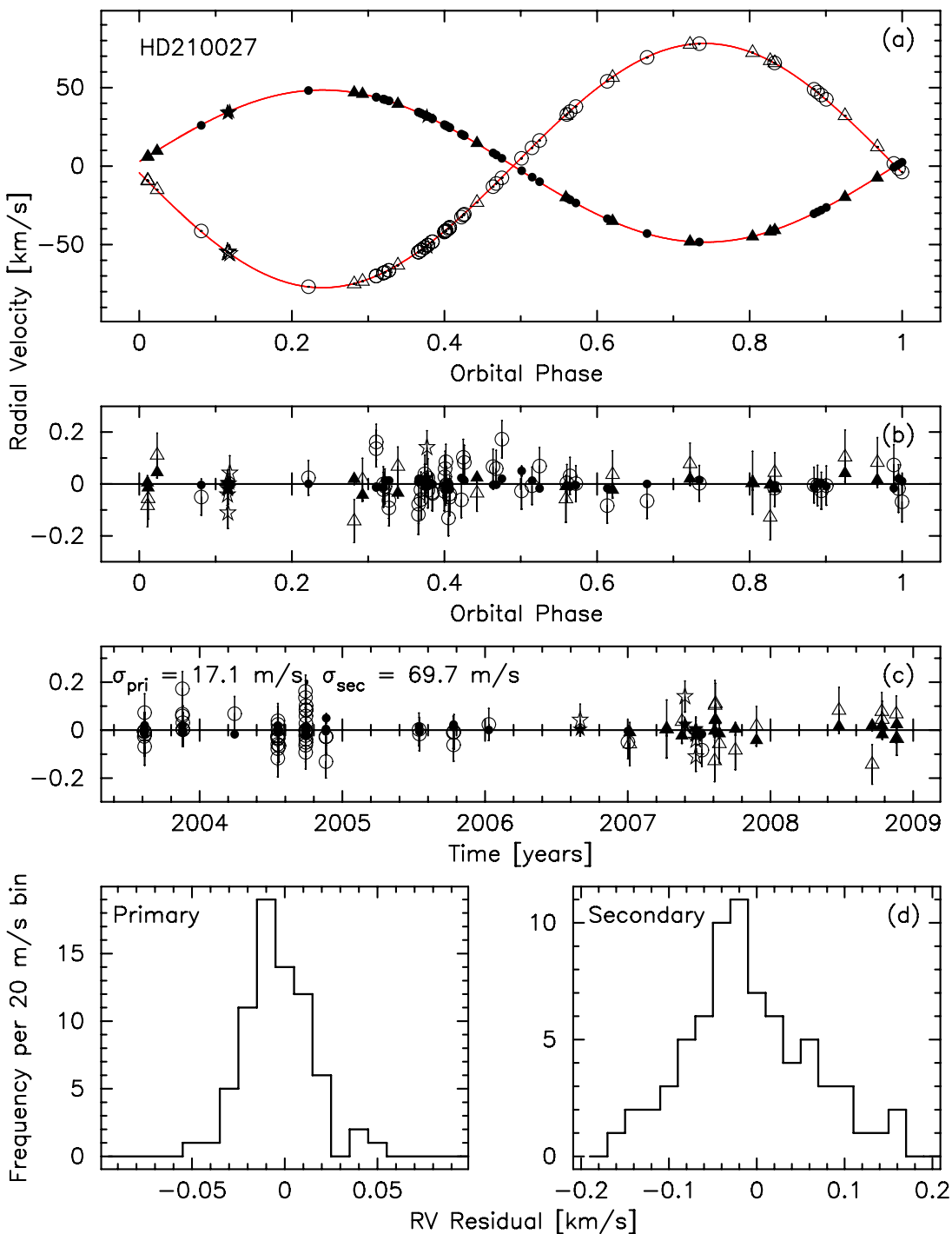

Figure 16. Observed and modeled radial velocities of HD210027 as a function of the orbital phase (a), their best-fit residuals as a function of the orbital phase (b) and time (c). The histograms of the residuals for the primary and secondary (d). The Keck I/HIRES is denoted with circles, Shane/CAT/Hamspec with triangles, and TNG/Sarg with stars.

(A color version of this figure is available in the online journal.)

et al. 1998; Mozurkewich et al. 1991)

$$
V_{\text {calibrated }}^{2}=V_{\text {measured }}^{2} / V_{\text {sys }}^{2},
$$

where

$$
V_{\text {sys }}^{2}=V_{\text {cal-measured }}^{2} / V_{\text {cal-expected }}^{2}
$$

and $V_{\text {cal-measured }}^{2}$ is a measured visibility and $V_{\text {cal-expected }}^{2}$ is an expected visibility of a calibrator given by Equation (9).

Visibilities of our targets were extracted from the NASA Exoplanet Science Institute's (NExSci) database of the PTI measurements. They were subsequently calibrated (see Table 3) using the excellent tools getCal (ver. 2.10.7) and wbCalib (ver. 1.7.4) provided by NExSci. The data reduction was carried out using the default parameters of wbCalib. The software provides $\left\{t_{i}, V_{i}^{2}, \sigma_{i}, \lambda_{i}, u_{i}, v_{i}\right\}$ where $t_{i}$ is the time of observation, $V_{i}^{2}$ is the calibrated visibility amplitude squared, $\sigma_{i}$ its error, $\lambda_{i}$ is the mean wavelength for the observation, and $\left(u_{i}, v_{i}\right)$ are the components of the projected baseline vector. We do not list them as they can be easily obtained using the archival PTI data and the available tools.

\section{LEAST-SQUARES FITTING TO THE COMBINED $\mathrm{RV} / V^{2}$ DATA SETS}

We determined the best-fit orbital parameters of the binaries in the standard way by minimizing the following function:

$$
\begin{aligned}
\chi^{2}= & \sum_{i=1}^{N}\left(V_{i}^{2}-\widehat{V}_{i}^{2}\right)^{2} / \sigma_{V_{i}^{2}}^{2}+\sum_{i=1}^{M}\left(\mathrm{RV} 1_{i}-\widehat{\mathrm{RV}} 1_{i}\right)^{2} / \sigma_{\mathrm{RV} 1_{i}}^{2} \\
& +\sum_{i=1}^{M}\left(\mathrm{RV}_{i}-\widehat{\mathrm{RV}} 2_{i}\right)^{2} / \sigma_{\mathrm{RV}_{2}}^{2}
\end{aligned}
$$

where $V_{i}^{2}$ and $\sigma_{V_{i}^{2}}, \mathrm{RV} 1_{i}$ and $\sigma_{\mathrm{RV} 1_{i}}$, and $\mathrm{RV} 2_{i}$ and $\sigma_{\mathrm{RV} 2_{i}}$ are, respectively, visibilities and their errors, RVs of the primary and their errors, and RVs of the secondary and their errors; $N$ and $M$ denote the number of measurements. The symbols with a hat denote the respective model values computed as described in the previous sections. The fit was carried out using the Levenberg-Marquardt algorithm for solving the minimization problem which was incorporated into our own software for 

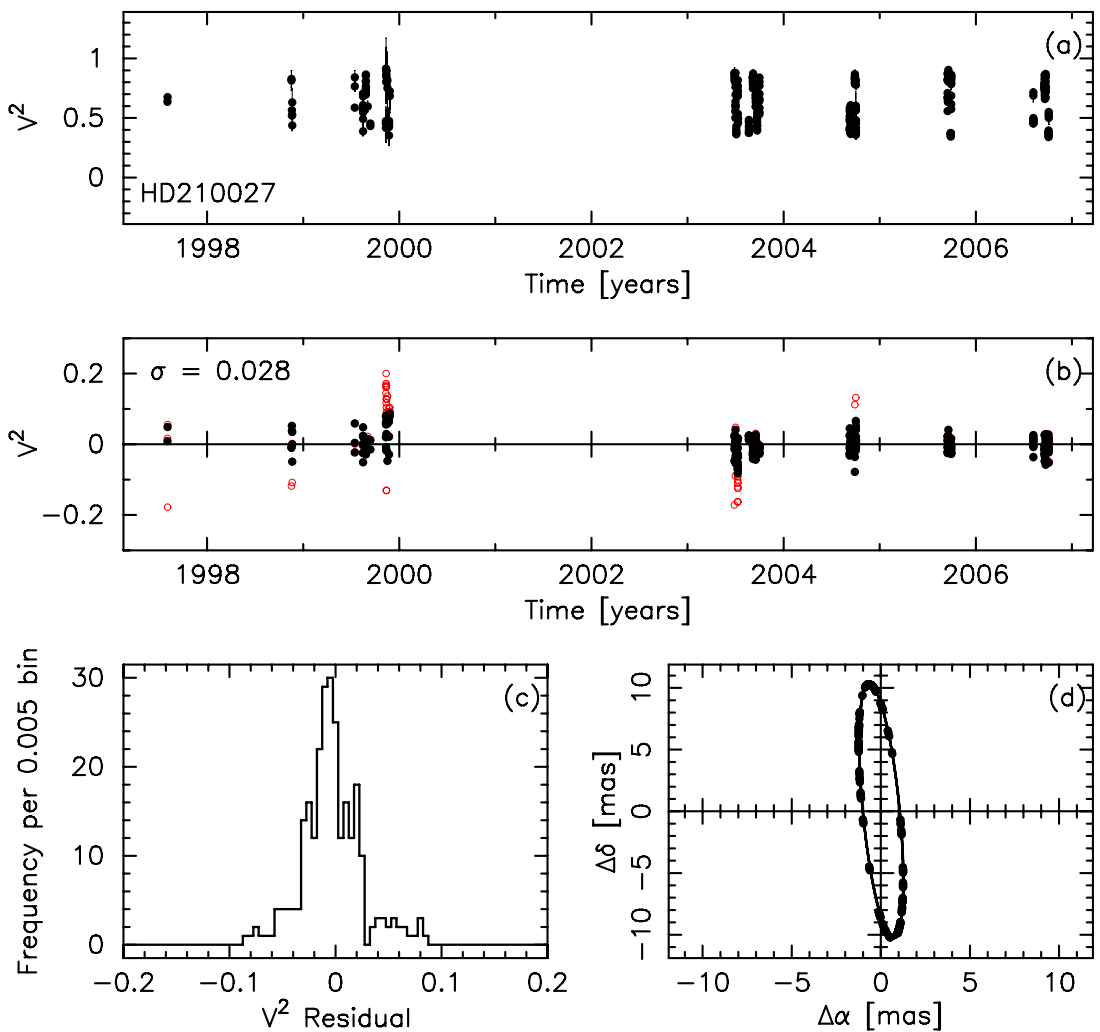

Figure 17. Visibility measurements of HD210027 as a function of time (a), their best-fit residuals as a function of time (b) and histogram (c). The measurements used to determine the best-fit orbital solution are denoted with filled circles. The corresponding orbital coverage and the relative orbit are shown in panel (d).

(A color version of this figure is available in the online journal.)

modeling and least-squares fitting of the visibilities and RVs. As mentioned above, the RV variations due to the tidal distortions of the components were modeled with the Wilson-Davinney's lc code (version from 2007) which was also incorporated into our software.

The least-squares fitting formalism allows one to compute the formal uncertainties (errors) of the best-fit parameters. However, such errors do not necessarily correspond to the true uncertainties of the parameters. In order to provide conservative estimates of these errors, we also accounted for the systematic errors in the modeling which are due to (1) the uncertainty in the projected baseline $(u, v),(2)$ the uncertainty in the mean wavelength $\lambda$, (3) the uncertainties in the diameters, $\theta_{j}$, of the calibrators (which affect the modeled visibility through Equation (20)) and the diameters of the binary components (which affect the modeled visibility through Equation (17)), and (4) the uncertainties in the parameters used to model the tidal RV effects. We assumed the following estimates for these uncertainties: (1) $0.01 \%$ in $(u, v)$, (2) $0.5 \%$ in $\lambda$, (3) $10 \%$ in the calibrator and binary components diameters, and (4) $10 \%$ in all the parameters from Table 2 except for the temperatures for which we assumed an uncertainty of 1\% for HD210027 and 2\% for the other targets; and for the metallicities we assumed an uncertainty of 0.05 dex. The errors of the parameters shown in Tables 4-6 include the contribution from the systematic errors.

Our procedure for deriving RVs results in slightly different zero points of the velocities for each data subset. These small differences are among others due to a mismatch, different for each component of a binary, of the synthetic templates and the real spectra. The synthetic templates, as already explained, are used in the first step of RV computations and the shifts are then carried over to the real disentangled component spectra. The other sources of the small zero-point differences are due to the use of different spectrographs and the accuracies with which the zero points of the template iodine cell transmission functions are known. The velocity shifts are determined together with the other parameters in the least-squares fits and are shown in Table 5. Even though the total formal errors of the gamma velocities are small, due to the above reasons it is unlikely that their realistic uncertainties are smaller than $\sim 0.1 \mathrm{~km} \mathrm{~s}^{-1}$.

The full statistical details of the best-fit solutions are given in Table 5 and the corresponding residuals are shown in Figures 8-17. For the RV data sets, we determined the additional $\mathrm{RV}$ errors added in the quadrature to the formal RV errors by performing separate orbital fits to the RVs alone and finding such additional RV errors which provide $\chi^{2} \approx 1$. For $V^{2}$ measurements, we used the errors as computed with wbCalib. However, by performing a preliminary fit to all the available $V^{2} \mathrm{~s}$ and RVs, we found those $V^{2}$ measurements that significantly deviate from the best-fit solutions. Such deviant measurements are most likely due to poor weather conditions and/or an errant behavior of the instrument. In the final fit, we did not use them. In figures showing visibility measurements (Figures 9, 11, 13, 15, and 17), they are denoted with open (red) circles. The resulting total $\chi^{2}$ values are close or very close to 1 which ensures one that the estimates of the errors of the parameters are realistic.

\subsection{Notes on the Individual Solutions}

The orbital parameters of the combined spectroscopic- astrometric solutions are shown in Tables 4 and 5 and the resulting physical parameters are shown in Table 6 . The masses expressed in the solar masses were derived with $G M_{\odot}=$ 

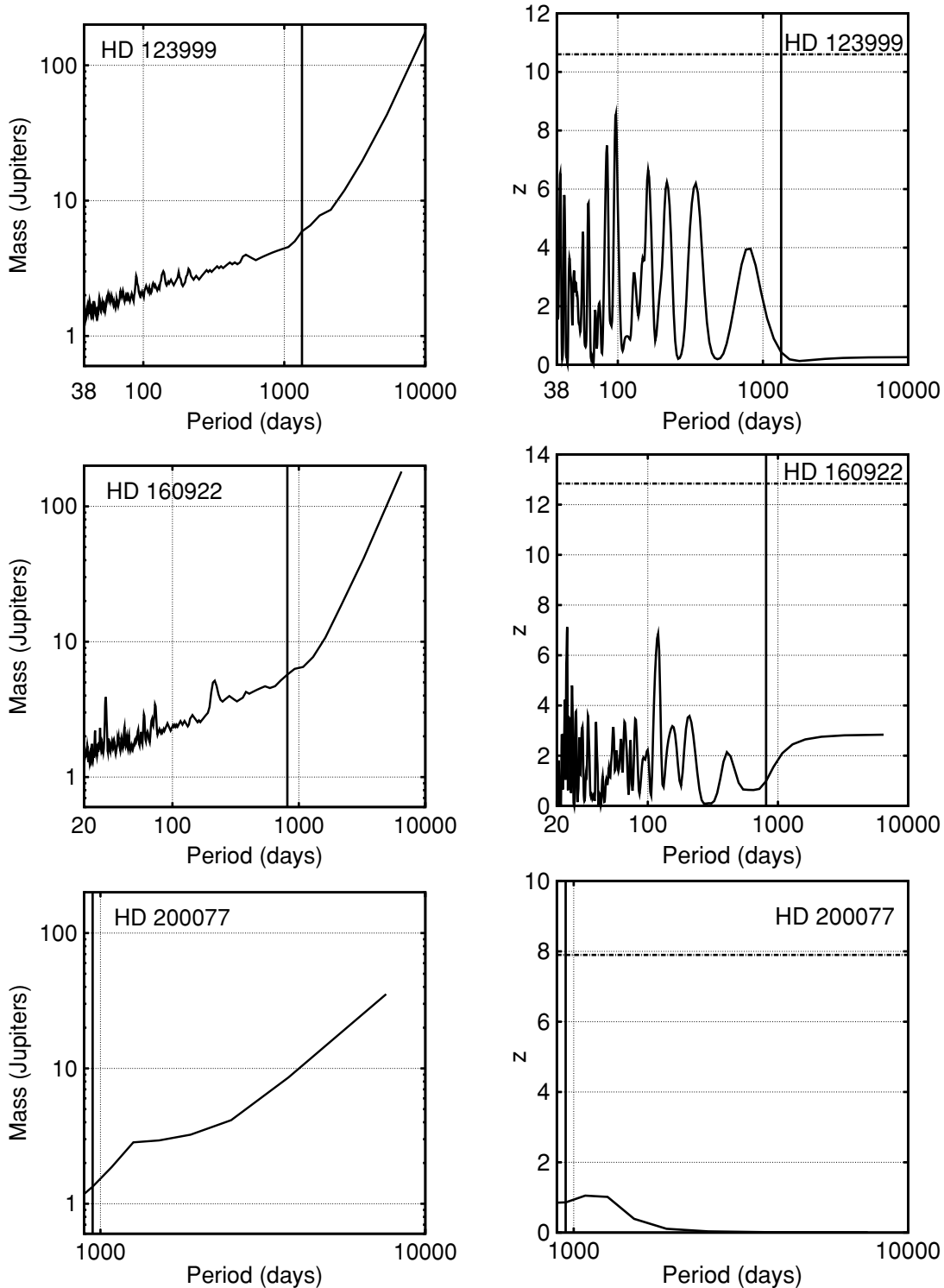

Figure 18. Circumbinary planet detection limits in the $\log P-\log M$ space (orbital period-mass; left panels) and periodograms (right panels). The solid horizontal line in the right panels is a planet detection limit corresponding to the $99 \%$ confidence level. The vertical line near the orbital period of 1000 days denotes the time span of the data set. The first stable planetary orbits have the periods of respectively 38, 20, and 890 days as calculated based on Holman \& Wiegert (1999).

$1.3271244017987 \times 10^{20} \mathrm{~m}^{3} \mathrm{~s}^{2}$, a value used in the DE405 JPL ephemerides. Let us note that the official value of $G$ recommended by The Committee on Data for Science and Technology (CODATA) is $(6.67428 \pm 0.00067) \times 10^{-11} \mathrm{~m}^{3} \mathrm{~kg}^{-1} \mathrm{~s}^{-2}$. Hence, $G$ is known with a fractional error of $0.01 \%$ and in consequence the mass of the Sun and the masses of stars expressed in absolute units are known with only such precision.

We also calculated limits to circumbinary planets for three new systems HD123999, HD160922, and HD200077. They were calculated as in Konacki et al. (2009) and are shown in Figure 18.

HD78418 (75 Cnc, HR 3626, HIP 44892; V = $5.98 \mathrm{mag}$, $K=4.37 \mathrm{mag}$ ) is a $\sim 19.4$ day period binary with a spectral type G5IV-V. The most recent spectroscopic solution is by de Medeiros \& Udry (1999) and is characterized by an RV rms of $461 \mathrm{~m} \mathrm{~s}^{-1}$. Our combined RV solution has an rms of 13.1 and $24.3 \mathrm{~m} \mathrm{~s}^{-1}$ for the primary and secondary, respectively. The binary was resolved with PTI by Lane \& Boden (1999) but a detailed spectroscopic-astrometric analysis was never published. Using the PTI data archive, we extracted $440 \mathrm{~V}^{2}$ measurements spanning 1999-2008 of which 415 were used in the final fit by adopting a cutoff at 0.1 for $V^{2}$ 's $O-C$. The masses of the components are $1.173 \pm 0.024 M_{\odot}(2.0 \%$ accuracy) and $1.011 \pm 0.021 M_{\odot}(2.1 \%$ accuracy $)$ for the primary and secondary, respectively. This is a far more accurate mass determination than the one from Lane \& Boden (1999). The masses and the absolute magnitudes in the $K$ and $H$ bands together with the Padova isochrones (Marigo et al. 2008) allow us to estimate the age of the system as about 2.5-4.0 Gyr (see Figures 19 and 20). For the isochrones, we adopted a metallicity of -0.09 (Nordström et al. 2008, 2004). The $J H K$ photometry comes from the Two Micron All Sky Survey (2MASS) catalog (Skrutskie et al. 2006). Let us note that over the years different values of the gamma velocity were obtained for HD78418 ranging from $12.3 \mathrm{~km} \mathrm{~s}^{-1}$ (Sanford 1922), $10.4 \mathrm{~km} \mathrm{~s}^{-1}$ (Beavers \& Salzer 1982) to below $10 \mathrm{~km} \mathrm{~s}^{-1}$ (de Medeiros \& Udry 1999). This could indicate a linear trend in RVs. However, we have not detected any linear trend in our RVs.

HD123999 (12 Boo, HR 5304, HIP 69226; V = $4.82 \mathrm{mag}$, $K=3.64)$ is a $\sim 9.6$ day period binary with spectral type F8IV. 

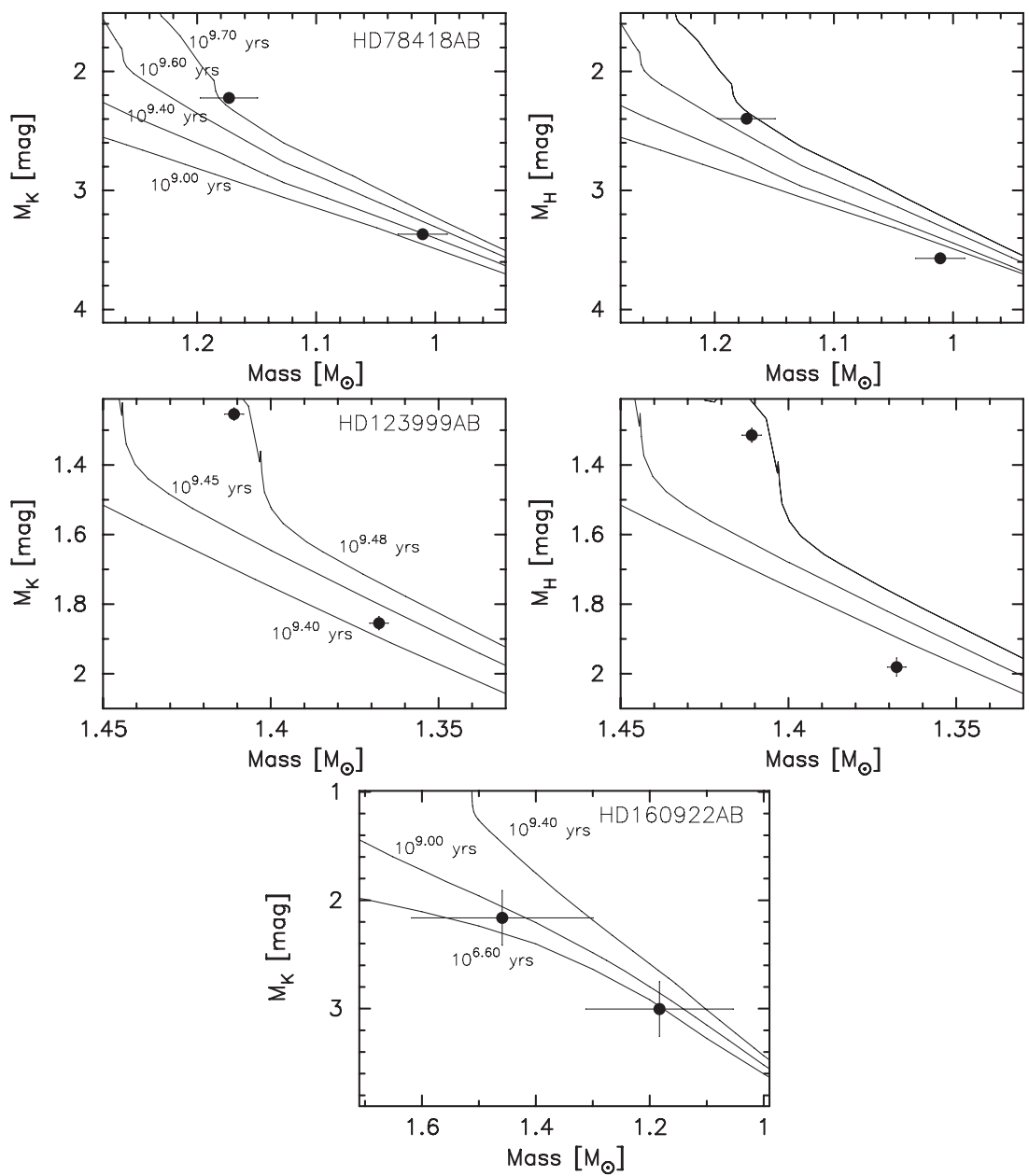

Figure 19. Isochrones in the mass-absolute magnitude plane for HD78418 (top), HD123999 (middle), and HD160922 (bottom) together with the corresponding best-fitting isochrones.

The latest spectroscopic orbit was published by Tomkin \& Fekel (2006). It has an RV rms of 180 and $100 \mathrm{~m} \mathrm{~s}^{-1}$ for the primary and secondary, respectively. Our combined RV data sets are characterized by an rms of 34.0 and $38.3 \mathrm{~m} \mathrm{~s}^{-1}$ which is due to somewhat wide spectral lines of the stars. The binary was resolved and studied with PTI by Boden et al. (2000) and most recently by Boden et al. (2005) who obtained a combined spectroscopic-astrometric solution using the PTI $V^{2}$ measurements collected in the years 1998-2004. We extracted $374 V^{2}$ measurements spanning 1999-2008 and used 346 in the final fit (a cutoff at 0.09 for $V^{2}$ 's $O-C$ ). The masses of the components are $1.4109 \pm 0.0028 M_{\odot}(0.2 \%$ accuracy $)$ and $1.3677 \pm 0.0028 M_{\odot}(0.2 \%$ accuracy $)$ for the primary and secondary, respectively. These values are consistent with those by Boden et al. (2005) but about two times more accurate. The RVs used by Boden et al. (2005) were characterized by an rms of 400 and $490 \mathrm{~m} \mathrm{~s}^{-1}$. Assuming a solar metallicity as Boden et al. (2005), the estimated age for the system is 2.5-2.9 Gyr. Note that as in Boden et al. (2005), the secondary appears to be younger than the primary. Our smaller error bars for the masses and absolute $K$ - and $H$-band magnitudes make this discrepancy even more apparent. The $J H K$ photometry comes from Boden et al. (2000).

HD160922 ( $\omega$ Dra, HR 6596, HIP 86201; V = $4.90 \mathrm{mag}$, $K=3.62 \mathrm{mag}$ ) is a $\sim 5.3$ day period binary with a spectral type F5V. An improved spectroscopic solution was recently published by Fekel et al. (2009). It is characterized by an RV rms of $190 \mathrm{~m} \mathrm{~s}^{-1}$. Our combined RV solution has an rms of 37.9 and $31.9 \mathrm{~m} \mathrm{~s}^{-1}$ for the primary and secondary, respectively. This somewhat lower RV precision is due to relatively wide spectral lines of the components. The PTI archive provides 62 never published $V^{2}$ measurements of which 61 were used in the final fit ( $V^{2}$,s $O-C$ cutoff at 0.08 ). We have obtained a spectroscopic-astrometric orbital solution with a small but statistically significant eccentricity $(0.00220 \pm 0.00031)$ which is in agreement with Fekel et al. (2009). However, they have decided to adopt a circular solution after all. The masses of the components are $1.46 \pm 0.16 M_{\odot}(11 \%$ accuracy $)$ and $1.18 \pm 0.13 M_{\odot}(11 \%$ accuracy $)$ for the primary and secondary, respectively. The masses are derived for the first time for this system but their accuracy is poor due to a limited number of $V^{2}$ measurements and the near face-on orbital configuration. Based on the derived masses and the $K$-band absolute magnitudes, we estimate the age of the system as 0.004-2.5 Gyr; a wide range due to large error bars both in the masses and the $K$-band magnitudes. For the isochrones, we adopted a solar metallicity (Nordström et al. 2008, 2004). The $J H K$ photometry comes from the 2MASS catalog.

HD200077 (HIP 103641; V = $6.57 \mathrm{mag}, K=5.12 \mathrm{mag}$ ) is a $\sim 112.5$ day period binary with a spectral type G0V. The spectroscopic orbital solution for both components was for the first time derived by Goldberg et al. (2002). Their RVs are 

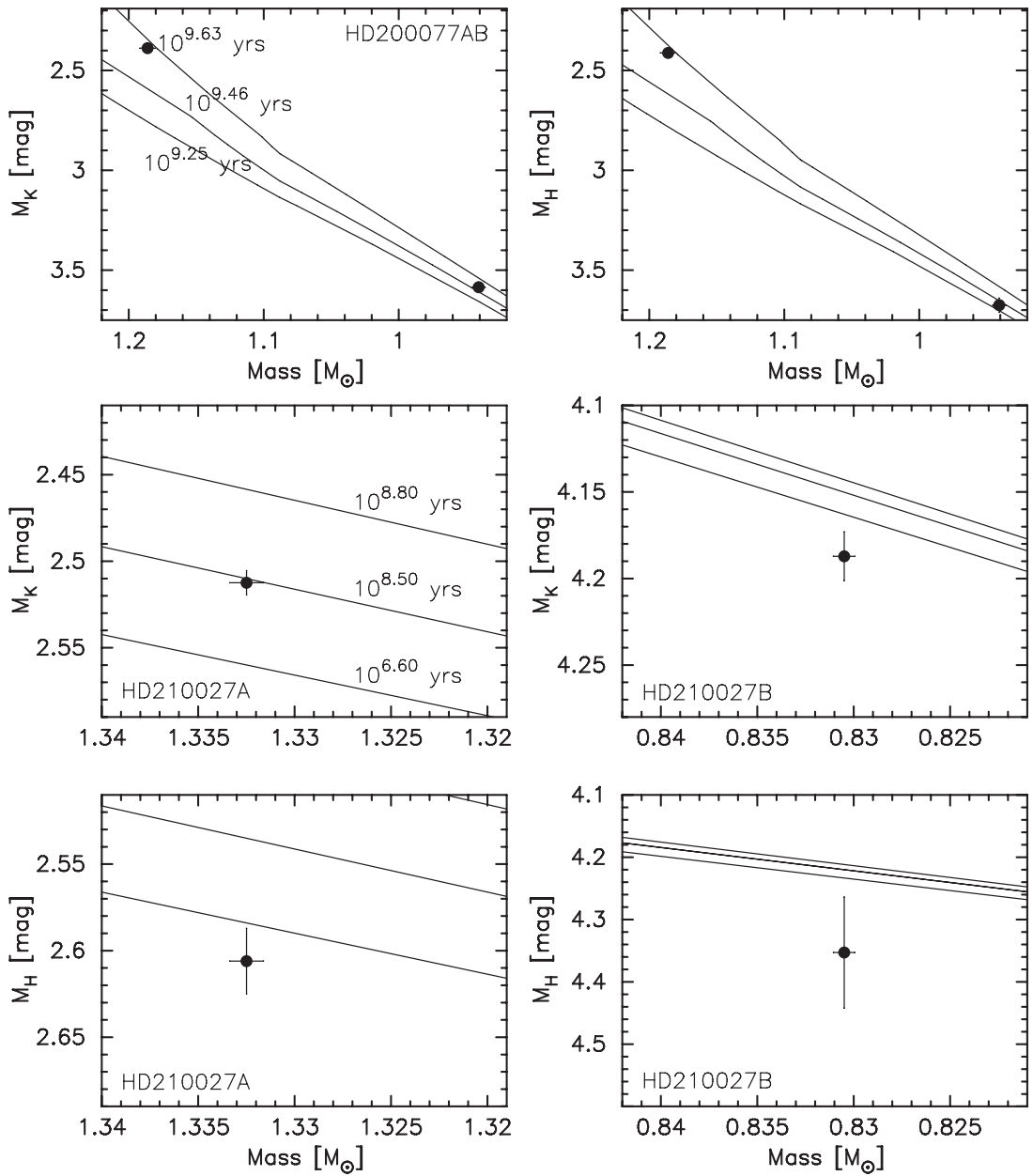

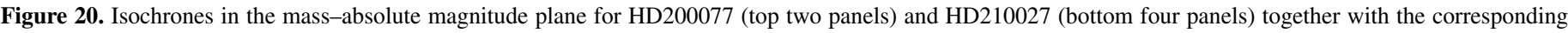
best-fitting isochrones. In the case of HD210027, their companions are shown separately so that it is easier to compare the error bars with the isochrones.

characterized by an rms of 600 and $2150 \mathrm{~m} \mathrm{~s}^{-1}$ for the primary and secondary, respectively. Our combined RV solution has an rms of, respectively, 8.5 and $30.4 \mathrm{~m} \mathrm{~s}^{-1}$. The binary was resolved with PTI but this has never been published before. The PTI archive provides $395 V^{2}$ measurements spanning 2001-2007 of which 329 were used in the final fit ( $V^{2}$ 's $O-C$ cutoff at 0.09). The masses of the components derived for the first time are $1.1860 \pm 0.0057 M_{\odot}(0.48 \%$ accuracy $)$ and $0.9407 \pm 0.0049 M_{\odot}(0.52 \%$ accuracy $)$ for the primary and secondary, respectively. Based on the masses, $K$ - and $H$-band absolute magnitudes, the estimated age is $2.9-4.3$ Gyr. For the isochrones, we adopted a metallicity of -0.14 (Nordström et al. $2008,2004)$. The $J H K$ photometry comes from the 2MASS catalog.

HD210027 ( $\mathrm{Peg}, 24$ Peg, HR 8430, HIP 109176; V= 3.76 mag, $K=2.56$ ) is a $\sim 10.2$ day period binary with spectral types F5V/G8V. It is one of the first SB2s resolved with PTI. The most recent orbital solution is a combined spectroscopic-astrometric solution by Boden et al. (1999). It is based on the PTI $V^{2}$ measurements from 1997 and the archival RV measurements from Fekel \& Tomkin (1983) which are characterized by an rms of about 600 and $700 \mathrm{~m} \mathrm{~s}^{-1}$ for the primary and secondary. Our combined RV solution has an rms of, respectively, 17.1 and $69.7 \mathrm{~m} \mathrm{~s}^{-1}$. The PTI archive provides $299 \mathrm{~V}^{2}$ measurements spanning 1997-2006 of which 266 were used in the final fit ( $V^{2}$,s $O-C$ cutoff at 0.088 ). The masses of the components are $1.33249 \pm 0.00086 M_{\odot}(0.065 \%$ accuracy $)$ and $0.83050 \pm$ $0.00055 M_{\odot}(0.066 \%$ accuracy $)$ for the primary and secondary, respectively. This is about a 20 times more accurate mass determination than the one from Boden et al. (1999) and constitutes the most accurate mass measurement for a normal star. Based on the masses, $K$ - and $H$-band absolute magnitudes, the estimated age is 4-663 Myr with $i$ sin agreement with Morel et al. (2000). For the isochrones, we adopted a solar metallicity (Morel et al. 2000). The JHK photometry comes from Bouchet et al. (1991). Note that our orbital solution has a small but statistically significant eccentricity $(0.001764 \pm$ $0.000063)$. This combined with the systems' young age may prove useful in studying the tidal history of HD210027.

\section{DISCUSSION}

There are a few ways to determine accurate masses of normal stars. Perhaps the most classic one is through absolute astrometric orbits of both components of a binary. However, this constitutes a challenging measurement and in practice masses are typically derived using diverse data sets, e.g., (1) by combining RVs, relative astrometry, and parallax for singlelined spectroscopic binaries, (2) as in this paper by combining relative astrometry and RVs for double-lined spectroscopic binaries, and (3) by combining light curves and RVs for eclipsing double-lined binaries. The last method is the most useful one as 
Table 6

Physical Parameters for HD78418, HD123999, HD160922, HD200077, and HD210027

\begin{tabular}{|c|c|c|c|}
\hline Star & Parameter & Primary & Secondary \\
\hline \multirow[t]{7}{*}{ HD78418 } & Semimajor axis, $a_{1,2}(\mathrm{AU})$ & $0.084874(11)$ & $0.098526(21)$ \\
\hline & $M \sin ^{3} i\left(M_{\odot}\right)$ & $0.191350(50)$ & $0.164836(45)$ \\
\hline & Mass, $M\left(M_{\odot}\right)$ & $1.173(24)$ & $1.011(21)$ \\
\hline & $M_{K, 2 \mathrm{MASS}}(\mathrm{mag})$ & $2.223(16)$ & $3.367(18)$ \\
\hline & $M_{H, 2 \mathrm{MASS}}(\mathrm{mag})$ & $2.397(37)$ & $3.570(45)$ \\
\hline & Parallax, $\kappa$ (mas) & & $32.004(52)$ \\
\hline & Distance, $d(\mathrm{pc})$ & & $31.246(51)$ \\
\hline \multirow[t]{7}{*}{ HD123999 } & Semimajor axis, $a_{1,2}(\mathrm{AU})$ & $0.061193(10)$ & $0.063125(13)$ \\
\hline & $M \sin ^{3} i\left(M_{\odot}\right)$ & $1.21467(33)$ & $1.17749(33)$ \\
\hline & Mass, $M\left(M_{\odot}\right)$ & $1.4109(28)$ & $1.3677(28)$ \\
\hline & $M_{K, 2 \mathrm{MASS}}(\mathrm{mag})$ & $1.254(18)$ & $1.855(19)$ \\
\hline & $M_{H, 2 \mathrm{MASS}}(\mathrm{mag})$ & $1.314(20)$ & $1.981(26)$ \\
\hline & Parallax, $\kappa$ (mas) & & $27.917(44)$ \\
\hline & Distance, $d(\mathrm{pc})$ & & $35.820(57)$ \\
\hline \multirow[t]{7}{*}{ HD160922 } & Semimajor axis, $a_{1,2}(\mathrm{AU})$ & $0.036728(16)$ & $0.045306(17)$ \\
\hline & $M \sin ^{3} i\left(M_{\odot}\right)$ & $0.160409(88)$ & $0.130039(80)$ \\
\hline & Mass, $M\left(M_{\odot}\right)$ & $1.46(16)$ & $1.18(13)$ \\
\hline & $M_{K, 2 \mathrm{MASS}}(\mathrm{mag})$ & $2.16(25)$ & $3.00(25)$ \\
\hline & $M_{H, 2 \mathrm{MASS}}(\mathrm{mag})$ & $\ldots$ & \\
\hline & Parallax, $\kappa$ (mas) & & $42.29(27)$ \\
\hline & Distance, $d(\mathrm{pc})$ & & $23.65(15)$ \\
\hline \multirow[t]{7}{*}{ HD200077 } & Semimajor axis, $a_{1,2}(\mathrm{AU})$ & $0.25945(74)$ & $0.32712(95)$ \\
\hline & $M \sin ^{3} i\left(M_{\odot}\right)$ & $0.8009(34)$ & $0.6352(30)$ \\
\hline & Mass, $M\left(M_{\odot}\right)$ & $1.1861(57)$ & $0.9407(49)$ \\
\hline & $M_{K, 2 \mathrm{MASS}}(\mathrm{mag})$ & $2.389(24)$ & $3.585(25)$ \\
\hline & $M_{H, 2 \text { MASS }}(\mathrm{mag})$ & $2.412(23)$ & $3.675(36)$ \\
\hline & Parallax, $\kappa$ (mas) & & $24.640(32)$ \\
\hline & Distance, $d(\mathrm{pc})$ & & $40.571(52)$ \\
\hline \multirow[t]{7}{*}{ HD210027 } & Semimajor axis, $a_{1,2}(\mathrm{AU})$ & $0.0457446(37)$ & $0.073395(15)$ \\
\hline & $M \sin ^{3} i\left(M_{\odot}\right)$ & $1.31190(29)$ & $0.81767(22)$ \\
\hline & Mass, $M\left(M_{\odot}\right)$ & $1.33249(86)$ & $0.83050(55)$ \\
\hline & $M_{K, 2 \mathrm{MASS}}(\mathrm{mag})$ & $2.5125(70)$ & $4.187(14)$ \\
\hline & $M_{H, 2 \mathrm{MASS}}(\mathrm{mag})$ & $2.606(19)$ & $4.353(89)$ \\
\hline & Parallax, $\kappa$ (mas) & & $86.70(14)$ \\
\hline & Distance, $d(\mathrm{pc})$ & & $11.534(19)$ \\
\hline
\end{tabular}

Note.

a The numbers in parentheses are the $1 \sigma$ errors in the last digits quoted.

it not only provides the most accurate masses due to a convenient edge-on geometry (note that the masses are derived from their respective $M \sin ^{3} i$ ) but also enables one to determine the radii of stars.

In a recent review, Torres et al. (2009) collect 118 detached binary stars (including 94 eclipsing) with the most accurate mass determinations in the literature. These are denoted with open circles in Figure 21. Even though our targets are not eclipsing and the orbital inclinations and their errors have a significant impact on the precision in masses, for two stars HD123999 and HD210027 we have obtained more accurate mass determinations than for any of the stars from Torres et al. (2009). The accuracies of the masses of these two binaries are in the precision range covered by only close double neutron star systems characterized with radio pulsar timing. In particular, the masses for HD210027 rival in precision the mass determination of the components of the relativistic double pulsar system PSR J0737-3039 (Nice et al. 2008). If our targets were all eclipsing and the accuracy in masses was limited by our RVs alone, the accuracy would be in the range $0.02 \%-0.42 \%$ (the fractional accuracy of $M \sin ^{3} i$ ). The lower limit of this range is equal to the mass accuracy of PSR B1913+16 which has the most accurate mass determination for a body outside the solar system

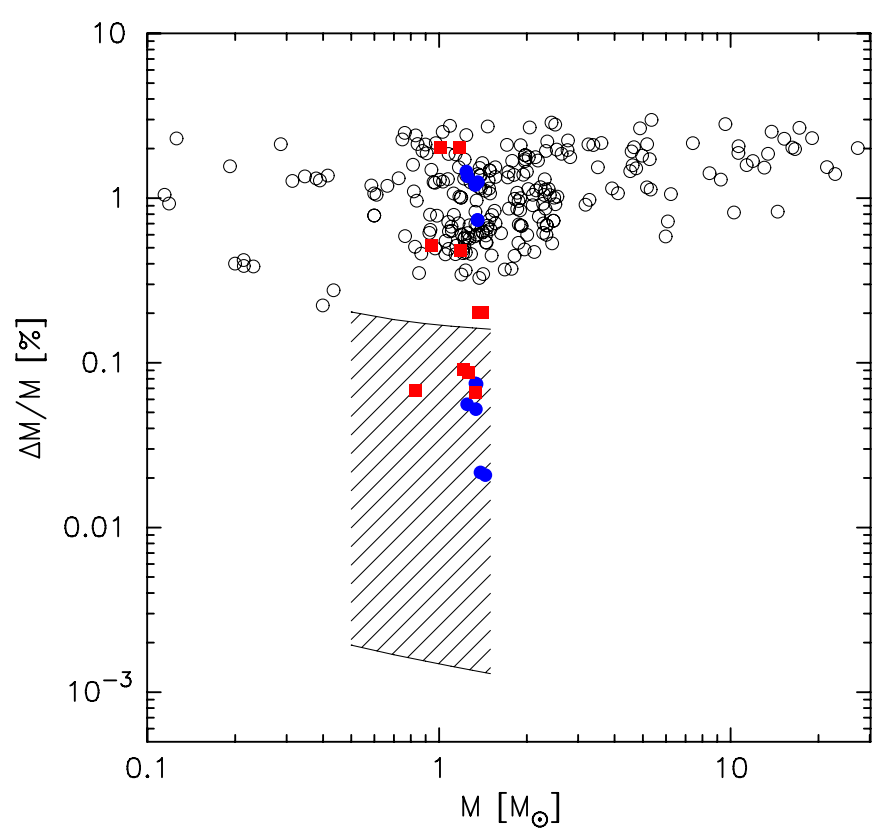

Figure 21. Fractional accuracy in masses for the binary stars with the most accurate mass determinations in the literature. Open circles denote the binaries from a recent review by Torres et al. (2009). Filled (blue) circles denote the double neutron star systems B1913+16, B2127+11C, B1534+12, J0737-3039, $\mathrm{J} 1756-2251$, and J1906+0746 characterized with radio pulsar timing (Nice et al. 2008). Filled (red) rectangles denote the masses for HD78418, HD123999, HD200077, and HD210027 from this paper and a mass determination for AI Phe from our recent work (Hełminiak et al. 2009). The hatched area is the expected precision in masses for double-lined eclipsing binary stars assuming a mass range of the components of $0.5-1.5 M_{\odot}$, orbital period range of 3-23 days, orbital inclination range of $85^{\circ}-90^{\circ}$, inclination's error range of $0.05-0.3$, and radial velocity amplitudes' error range of $1-31 \mathrm{~m} \mathrm{~s}^{-1}$.

(A color version of this figure is available in the online journal.)

(Nice et al. 2008). In fact, if we adopt our RV precision and use the achievable from the ground precision in the orbital inclination angle for eclipsing binaries, we can expect to obtain masses with a fractional precision of $0.001 \%$ (see Figure 21). Clearly, our RV technique for double-lined and eclipsing binary stars opens an exciting opportunity for deriving masses of stars (and other parameters) with an unprecedented precision. These combined with parallax measurements from, e.g., the planned GAIA astrometric mission and hopefully accurate abundance determinations should produce an outstanding set of parameters to test models of the stellar structure and evolution.

We thank the California and Carnegie Exoplanet Search team, and Geoff Marcy in particular, for allowing us access to their precision velocimetry tools at Lick Observatory. This work benefits from the efforts of the PTI collaboration members who have each contributed to the development of an extremely reliable observational instrument. We thank PTI's night assistant Kevin Rykoski for his efforts to maintain PTI in excellent condition and operating PTI. This research has made use of the Simbad database, operated at CDS, Strasbourg, France. M.W.M. acknowledges support from the Townes Fellowship Program. M.K. is supported by the Foundation for Polish Science through a FOCUS grant and fellowship and by the Polish Ministry of Science and Higher Education through grant N203 005 32/ 0449. Part of the algorithms used in this analysis were developed during the SIM Double Blind Test, under JPL contract 1336910. This research has made use of the Simbad database, operated at 
CDS, Strasbourg, France. The observations on the TNG/Sarge have been funded by the Optical Infrared Coordination network (OPTICON), a major international collaboration supported by the Research Infrastructures Programme of the European Commissions Sixth Framework Programme. This publication makes use of data products from the Two Micron All Sky Survey, which is a joint project of the University of Massachusetts and the Infrared Processing and Analysis Center/California Institute of Technology, funded by the National Aeronautics and Space Administration and the National Science Foundation. This research was supported in part by the National Science Foundation under grant No. PHY05-51164.

Facilities: Keck:I (HIRES), TNG (Sarg), Shane (Hamspec), PO:PTI

\section{REFERENCES}

Bagnuolo, W. G., Jr., \& Gies, D. R. 1991, ApJ, 376, 266

Beavers, W. I., \& Salzer, J. J. 1982, PASP, 94, 356

Boden, A. F. 2000, in Principles of Long Baseline Stellar Interferometry, Proc. 1999 Michelson Summer School, ed. P. Lawson (JPL Publication 00-009 07/00; Pasadena, CA: JPL), 9 (http://olbin.jpl.nasa.gov/iss1999/ coursenotes.html)

Boden, A. F., Colavita, M. M., van Belle, G. T., \& Shao, M. 1998, Proc. SPIE, 3350, 872

Boden, A. F., Creech-Eakman, M. J., \& Queloz, D. 2000, ApJ, 536, 880

Boden, A. F., Torres, G., \& Hummel, C. A. 2005, ApJ, 627, 464

Boden, A. F., et al. 1999, ApJ, 515, 356

Bouchet, P., Schmider, F. X., \& Manfroid, J. 1991, A\&AS, 91, 409

Campbell, W. W. 1898, ApJ, 8, 123

Campbell, W. W. 1900, ApJ, 11, 259

Campbell, W. W. 1916, Lick Obs. Bull., 9, 30

Campbell, W. W., \& Curtis, H. D. 1905, Lick Obs. Bull., 3, 136

Campbell, W. W., Moore, J. H., Wright, W. H., \& Duncan, J. C. 1911, Lick Obs. Bull., 6, 140

Colavita, M. M. 1999, PASP, 111, 111

Colavita, M. M., et al. 1999, ApJ, 510, 505

de Medeiros, J. R., \& Udry, S. 1999, A\&A, 346, 532

Eaton, J. A. 2008, ApJ, 681, 562

Endl, M., Kürster, M., \& Els, S. 2000, A\&A, 362, 585

Fekel, F. C., \& Tomkin, J. 1983, PASP, 95, 1000

Fekel, F. C., Tomkin, J., \& Williamson, M. H. 2009, AJ, 137, 3900

Goldberg, D., Mazeh, T., Latham, D. W., Stefanik, R. P., Carney, B. W., \& Laird, J. B. 2002, AJ, 124, 1132

Hełminiak, K. G., Konacki, M., Ratajczak, M., \& Muterspaugh, M. 2009, MNRAS, 400, 969

Holman, M. J., \& Wiegert, P. A. 1999, AJ, 117, 621

Kaplan, G. H., Hughes, J. A., Seidelmann, P. K., Smith, C. A., \& Yallop, B. D. 1989, AJ, 97, 1197
Konacki, M. 2005, ApJ, 626, 431

Konacki, M. 2009, in IAU Symp. 253, Transiting Planets, ed. F. Pont, D. Sasselov, \& M. Holman (Cambridge: Cambridge Univ. Press), 141

Konacki, M., \& Lane, B. F. 2004, ApJ, 610, 443

Konacki, M., Muterspaugh, M. W., Kulkarni, S. R., \& Hełminiak, K. G 2009, ApJ, 704, 513

Konacki, M., Torres, G., Sasselov, D. D., \& Jha, S. 2003, ApJ, 597, 1076

Kopal, Z. 1980, Ap\&SS, 70, 329

Kopeikin, S. M., \& Ozernoy, L. M. 1999, ApJ, 523, 771

Kovalevsky, J. 1995, Modern Astrometry (Astron. and Astrophys. Lib.; Vol. XIV New York: Springer)

Kurucz, R. L. 1995, in ASP Conf. Ser. 78, Astrophysical Applications of Powerful New Databases, ed. S. J. Adelman \& W. L. Wiese (San Francisco, CA: ASP), 205

Lane, B. F., \& Boden, A. F. 1999, in ASP Conf. Ser. 194, Working on the Fringe: Optical and IR Interferometry from Ground and Space, ed. S. Unwin \& R. Stachnik (San Francisco, CA: ASP), 51

Marcy, G. W., \& Butler, R. P. 1992, PASP, 104, 270

Marigo, P., Girardi, L., Bressan, A., Groenewegen, M. A. T., Silva, L., \& Granato, G. L. 2008, A\&A, 482, 883

Mazeh, T. 2008, in EAS Publications Ser. 29, Tidal Effects in Stars, Planets, and Disks, ed. M.-J. Goupil \& J.-P. Zahn (Les Ulis: EDP Sciences), 1

Mazeh, T., Simon, M., Prato, L., Markus, B., \& Zucker, S. 2003, ApJ, 599, 1344

Morel, P., Morel, C., Provost, J., \& Berthomieu, G. 2000, A\&A, 354, 636

Mozurkewich, D., et al. 1991, AJ, 101, 2207

Nice, D. J., Stairs, I. H., \& Kasian, L. E. 2008, in AIP Conf. Proc. 983, 40 Years of Pulsars: Millisecond Pulsars, Magnetars and More, ed. C. G. Bassa et al (Melville, NY: AIP), 453

Nordström, B., et al. 2004, A\&A, 418, 989

Nordström, B., et al. 2008, VizieR Online Data Catalog, 5117, 0

Pickering, E. C. 1890, Observatory, 13, 80

Plummer, H. C. K., Wright, W. H., \& Turner, A. B. 1908, Lick Obs. Bull., 5, 21

Pourbaix, D., et al. 2004, A\&A, 424, 727

Sanford, R. F. 1922, ApJ, 55, 30

Skrutskie, M. F., et al. 2006, AJ, 131, 1163

Sterne, T. E. 1941, Proc. Natl Acad. Sci., 27, 168

Tomkin, J., \& Fekel, F. C. 2006, AJ, 131, 2652

Torres, G., Andersen, J., \& Gimenez, A. 2010, A\&ARv, 18, 67

Valenti, J. A., Butler, R. P., \& Marcy, G. W. 1995, PASP, 107, 966

van de Kamp, P. 1967, Principles of Astrometry (San Francisco, CA: Freeman)

Vogel, H. C. 1890a, Astron. Nachr., 123, 289

Vogel, H. C. 1890 b, PASP, 2,27

Vogel, H. C. 1890c, MNRAS, 50, 239

Vogel, H. C. 1891, MNRAS, 52, 87

Vogel, H. C. 1900, ApJ, 11, 393

Wilson, R. E. 1979, ApJ, 234, 1054

Wilson, R. E., \& Devinney, E. J. 1971, ApJ, 166, 605

Wilson, R. E., \& Sofia, S. 1976, ApJ, 203, 182

Zucker, S., \& Alexander, T. 2007, ApJ, 654, L83

Zucker, S., \& Mazeh, T. 1994, ApJ, 420, 806

Zucker, S., Mazeh, T., Santos, N. C., Udry, S., \& Mayor, M. 2004, A\&A, 426, 695 